\title{
The FORUM End-to-End Simulator project: architecture and results
}

\author{
Luca Sgheri $^{1}$, Claudio Belotti ${ }^{2}$, Maya Ben-Yami ${ }^{3}$, Giovanni Bianchini ${ }^{2}$, Bernardo Carnicero \\ Dominguez $^{3}$, Ugo Cortesi ${ }^{4}$, William Cossich ${ }^{5}$, Samuele Del Bianco ${ }^{4}$, Gianluca Di Natale ${ }^{2}$, \\ Tomás Guardabrazo ${ }^{6}$, Dulce Lajas ${ }^{3}$, Tiziano Maestri ${ }^{5}$, Davide Magurno ${ }^{5}$, Hilke Oetjen ${ }^{3}$, \\ Piera Raspollini ${ }^{4}$, and Cristina Sgattoni ${ }^{1}$ \\ ${ }^{1}$ IAC - CNR, Via Madonna del Piano 10, 50019 Sesto Fiorentino (FI), Italy \\ ${ }^{2}$ INO - CNR, Via Madonna del Piano 10, 50019 Sesto Fiorentino (FI), Italy \\ ${ }^{3}$ ESA - ESTEC, Keplerlaan 1, 2201 AZ Noordwijk, The Netherlands \\ ${ }^{4}$ IFAC - CNR, Via Madonna del Piano 10, 50019 Sesto Fiorentino (FI), Italy \\ ${ }^{5}$ Università di Bologna - Dipartimento di Fisica e Astronomia, Viale Berti Pichat 6/2, 40126 Bologna, Italy \\ ${ }^{6}$ DEIMOS Space S.L.U., Ronda de Pte. 19, 28760 Tres Cantos - Madrid, Spain
}

Correspondence: Luca Sgheri (1.sgheri@iac.cnr.it)

\begin{abstract}
.
FORUM (Far-infrared Outgoing Radiation Understanding and Monitoring) will flight as the 9th ESA's Earth Explorer mission, and an End-to-End Simulator (E2ES) has been developed as a support tool for the mission selection process and the subsequent development phases. The current status of the FORUM E2ES project is presented, together with the characterization of the capabilities of a full physics retrieval code applied to FORUM data. We show how the instrument characteristics and the observed scene conditions impact on the spectrum measured by the instrument, accounting for the main sources of error related to the entire acquisition process, and the consequences on the retrieval algorithm. Both homogeneous and heterogeneous case studies are simulated in clear and cloudy conditions, validating the E2ES against two independent codes: KLIMA (clear sky) and SACR (cloudy sky). The performed tests show that the performance of the retrieval algorithm is compliant with the project requirements both in clear and cloudy conditions. The far infrared (FIR) part of the FORUM spectrum is shown to be sensitive to surface emissivity, in dry atmospheric conditions, and to cirrus clouds, resulting in improved performance of the retrieval algorithm in these conditions. The retrieval errors increase with increasing the scene heterogeneity, both in terms of surface characteristics and in terms of fractional cloud cover of the scene.
\end{abstract}

\section{Introduction}

FORUM (Far-infrared Outgoing Radiation Understanding and Monitoring, Palchetti et al. 2020) is a satellite mission selected in 2019 as the ninth ESA (European Space Agency) Earth Explorer. FORUM is conceived to fly in loose formation with IASI-NG (Infrared Atmospheric Sounder Interferometer - New Generation) on MetOp-SG-1A, and provide interferometric measurements in the 6.25-100 $\mu \mathrm{m}$ spectral interval encompassing the Far InfraRed (FIR) part of the spectrum (about 15- 
$100 \mu \mathrm{m}$ ) which is responsible for about $50 \%$ of the outgoing longwave flux (Harries et al., 2008) lost by our planet into space and it may be as large as $60 \%$ in polar regions.

Noteworthy, numerous satellite hyperspectral sounders are currently sampling the Mid InfraRed (MIR), such as AIRS (Chahine et al., 2006), IASI (Hilton et al., 2012) or CrIS (Han et al., 2013), but none of them is able to measure the Top of the Atmosphere (TOA) spectrum in the FIR. The unique contribution of the FORUM mission will allow to fill a major observational gap in the knowledge of the Earth energy budget and to study the role and the interactions among essential climate variables. The spectral characteristics of the incoming and outgoing radiation contain the fingerprints of surface, atmospheric and cloud processes such as those involving the water vapor and temperature profiles, ice cloud features and surface properties of polar and very dry regions. These components affect both the TOA energy budget and the energy distribution within the climate system. The outgoing longwave radiation, approximately from 100 to $2500 \mathrm{~cm}^{-1}$, is strongly dependent on surface temperature and emissivity, greenhouse gases concentration and cloud properties among others.

In particular, the spectral coverage and resolution of the FORUM interferometer are suitable to study the signatures of three key components of the climate system:

- Upper Troposphere Lower Stratosphere (UTLS) Water vapor: Clough and Iacono (1995); Sinha and Harries (1995); Brindley and Harries (1998) demonstrated that most of the cooling of the atmosphere occurs in the UTLS by means of rotational transitions of the water vapor molecules at FIR wavelengths. For the same band it was noted by Amato et al. (2002) that radiance sensitivity to water vapor changes is higher than in the MIR. Ridolfi et al. (2020) demonstrated the benefit of synergistic retrieval from simulated FORUM and IASI-NG measurements to provide improved L2 products among which UTLS water vapor profiles. Significant results on the retrieval of water vapor vertical profile and continuum coefficients have been obtained from the analysis of downwelling spectral radiances acquired in the far-infrared by the REFIR-PAD spectrometer at Dome-Concordia station in Antarctica, as reported by Palchetti et al. (2015) and Liuzzi et al. (2014), respectively. FORUM would thus be able to observe changes in the spectral signatures to assess seasonal and longer-term variations in UTLS water vapour and allow assessment of the underlying spectroscopy (ESA, 2019).

- Surface emissivity in polar and dry regions: Ice surface emissivity at FIR wavelengths has not been extensively validated (Chen et al., 2014) and most of the studies are based on theoretical modelling rather than measurements . Feldman et al. (2014) estimated the impact on wrong assumption of the spectral emissivity values at FIR on decadal average Artic surface temperature of the order of $2 \mathrm{~K}$. They also demonstrated the impact of surface emissivity on Artic-sea ice extent among others. Previous studies addressed the problem of simultaneous retrieval of surface temperature and emissivity by applying innovative physical inversion (Paul et al., 2012) and regularization (Masiello and Serio, 2013) schemes to IASI high spectral resolution radiances in the MIR. The FORUM mission will observe high-latitude scenes to retrieve a FIR surface emissivities dataset and possibly assess seasonal and inter-annual variability.

- Cirrus clouds: the role of ice clouds in regulating the climate has been recognized by many authors (e.g. Liou and Yang, 2016) despite the fact that thin ice clouds occurrence and properties remain uncertain especially in high latitude regions. This is one of the reason because their representation in GCMs still remains poor (IPCC, 2021). Recently, some authors 
(such as Maestri et al., 2019a; Saito et al., 2020; Di Natale et al., 2020) have shown the potentialities of exploiting FIR spectrally resolved radiances to refine ice clouds microphysical and optical properties. Maestri et al. (2019b) and Cossich et al. (2021) also showed the benefit of using FIR channels in combination with MIR ones to largely improve the performances of cloud identification and classification (i.e. between ice and liquid water phase) algorithms.

In the early stages of the mission development, an End-to-End Simulator (E2ES) has been devised as a tool for demonstrating proof-of-concept of the instruments and to evaluate the impact of instrument characteristics and scene conditions on the quality (precision, accuracy) of the retrieved products. The E2ES is composed of a chain of modules which simulates the whole process of measurement acquisition, propagating all the main sources of discrepancies conceivable in operative conditions through to the retrieved geophysical quantities. Thus, it is a much more complex process than the simple simulation and retrieval that can be obtained by a single code (convolving a high-resolution spectrum with an estimate of the instrumental lineshape). The aim is to be able to single out each possible source of error and assessing the sensitivity on both the level 1 (L1) and level 2 (L2) products. In the future, this simulator will act as the testbed for ESA's operational Level 2 development and also provide data for testing measurements in applications.

The E2ES has been a support tool in the selection process, and the development has been extended after the selection to add some additional features. The E2ES version considered in this paper is that representative of the instrument knowledge of the so-called Phase B1, i.e. the preliminary definition phase of a space mission, which extended up to December 2020. In Section 2 we briefly describe the FORUM mission, and the main targets. In Section 3 we present the architecture of the E2ES and the various modules of the execution chain. In Section 4 we introduce the selected scenes used for testing. In Section 5 we show the validation and assessment tests. Finally, we draw the conclusions in Section 6.

\section{The FORUM mission and role of the E2ES}

\subsection{The FORUM mission objectives}

The FORUM mission will provide an innovative observation of the distribution of a large part of the OLR by including the unmeasured FIR part of the spectrum. The measurements will allow to quantitatively evaluate the contribution of the FIR in the radiation balance, with the goal of reducing uncertainties in the prediction of climate models.

The L1 products of FORUM will constitute a highly accurate global dataset of FIR spectral upwelling radiances in multiple atmospheric conditions which is currently unavailable to the scientific community. The dataset aims at providing a benchmark for the evaluation of radiative routines in climate models through the use of satellite simulators (ESA, 2019). The objectives of the L1 FORUM radiance measurements also include: i) the validatation and refinement of the current spectroscopic properties (such as those concerning water vapor and in particular the continuum absorption, e.g., Serio et al. (2008), Mlawer et al. (2019)); ii) the evaluation of the level of accuracy of current radiative transfer codes in an under-explored part of the spectrum; iii) the characterization of the radiative signal in presence of high level clouds and the enhancement of the performances of the algorithms used in cirrus cloud identification from infrared measurements only (Maestri et al., 2019b). 

tropospheric water vapor (Ridolfi et al., 2020) and other greenhouse gases; ii) allow the derivation of ice cloud microphysical and optical properties (Maestri et al., 2019a; Saito et al., 2020) and the definition of new parametrizations for cirrus cloud radiative schemes (Di Natale et al., 2020; Bozzo et al., 2008); iii) provide surface emissivity in the FIR in polar and dry regions.

\subsection{FORUM requirements}

The FORUM satellite combines a Fourier transform spectrometer, namely the FORUM Sounding Instrument (FSI), and a FORUM Embedded Imager (FEI). The FEI measures a a radiance signal over a single band for a rectangular grid of $60 \times 60$ points, with a sampling step of $600 \mathrm{~m}$. The FSI measures the $100-1600 \mathrm{~cm}^{-1}$ spectrum, with a circular Field of View (FoV) with a diameter of $15 \mathrm{~km}$. The FSI FoV is centered with respect to the FEI grid since both instruments use the same telescope.

The requirements for the FORUM mission were defined from the scientific objectives of the mission in the framework of ESA project FORUMreq (see the FORUMreq final report available at: https://www.forum-ee9.eu). The complete sets of requirements for the FSI Level 1 (L1, i.e. the calibrated and geo-located radiances), Level 2 (L2, i.e. the retrieved geophysical quantities) and FEI L1 products are reported in Table 1, Table 2, Table 3, respectively. In this section, only the main specifications are discussed together with their driving scientific goals.

100 The FSI spectral range (100-1600 $\mathrm{cm}^{-1}$ ) was defined to cover most (96.7\%) of the spectrum of the Earth's infrared emission to space, with a spectral resolution of $0.5 \mathrm{~cm}^{-1}$ to identify the spectral signatures due to seasonal, and longer-term variations of water vapor in the upper troposphere - lower stratosphere (UTLS) range.

The requirements on precision were defined in terms of noise equivalent spectral radiance (NESR) and were set to meet the primary research objectives of FORUM: to identify FIR features of water vapor, thin cirrus clouds and surface properties.

One of the FORUM goals is to reduce uncertainty in climate predictions. The limits selected for absolute radiometric accuracy (ARA) allow to capture typical climate feedback signals associated with changes in surface temperature or perturbations in water vapor. Importantly, the goal level of accuracy $\left(0.1 \mathrm{~K}\right.$ in $\left.300-1100 \mathrm{~cm}^{-1}\right)$ will also allow the observations to be used, with confidence, as a benchmark against which future spectrally-resolved measurements can be compared (Wielicki et al., 2013).

110 Requirements on L2 products are realistically obtainable performances, derived by the instrument characteristics set by FSI requirements.

The area covered by the FEI field of view (FoV) is designed to include the FSI pixel (a circle of $7.5 \mathrm{~km}$ radius) and IASI-NG co-located measurements. A spatial sampling of $0.6 \mathrm{~km}$ is needed to detect clouds to which the FSI is sensitive.

\subsection{The role of the E2ES}

The key feature of the E2ES is the capability to introduce and single out possible causes for the discrepancies between the true and retrieved parameters. This applies both to the L1 products, i.e. the difference between the radiation reaching the instrument 
Table 1. FORUM FSI requirements

\begin{tabular}{|l|c|}
\hline FSI feature & Goal \\
\hline FSI Spectral coverage & $100-1600 \mathrm{~cm}^{-1}$ \\
\hline FSI spectral resolution (FWHM) & $0.5 \mathrm{~cm}^{-1}$ \\
\hline & $<0.2 \mathrm{~K}$ in $200-300 \mathrm{~cm}^{-1}$ \\
& $<0.1 \mathrm{~K}$ in $300-1100 \mathrm{~cm}^{-1}$ \\
FSI ARA $-3 \sigma$ & $<0.2 \mathrm{~K} \mathrm{in} 1100-1300 \mathrm{~cm}^{-1}$ \\
& $<1 \mathrm{~K} \mathrm{elsewhere}$ \\
\hline FSI NESR & $0.4 \mathrm{~mW} /\left(\mathrm{m}^{2} \mathrm{sr} \mathrm{cm}^{-1}\right)$ in $200-800 \mathrm{~cm}^{-1}$ \\
& $<1 \mathrm{~mW} /\left(\mathrm{m}^{2} \mathrm{sr} \mathrm{cm}^{-1}\right)$ elsewhere \\
\hline FSI frequency calibration & $1 \mathrm{ppm}$ \\
\hline FSI spatial sampling & $\leq 100 \mathrm{~km}$ \\
\hline
\end{tabular}

Table 2. FORUM FSI products requirements

\begin{tabular}{|l|l|}
\hline Product & Uncertainty requirement \\
\hline All-sky broadband spectral flux & $\begin{array}{l}\text { FORUM Level 1 FIR OLR extended to broadband with the Level 1 from IASI-NG, consis- } \\
\text { tent with independent broadband flux observations to within the radiance to flux uncertainty, } \\
\text { with minimal bias averaged over all scenes }\end{array}$ \\
\hline Water vapor profile & $\begin{array}{l}\text { Vertical profiles of water vapor concentration with } 15 \% \text { uncertainty at } 2 \mathrm{~km} \text { vertical resolu- } \\
\text { tion }\end{array}$ \\
\hline Surface emissivity & 0.01 in the $300-600 \mathrm{~cm}^{-1}$ spectral range for polar region on $50 \mathrm{~cm}^{-1}$ spectral grid $^{-20 \mathrm{~g} / \mathrm{m}^{2}}$ \\
\hline Ice water path (IWP) & $1 \mathrm{~km}$ \\
\hline Cloud Top Height (CTH) & $20 \%$ \\
\hline Particle size diameter & \\
\hline
\end{tabular}


Table 3. FORUM FEI requirements

\begin{tabular}{|l|l|}
\hline FEI feature & Requirement \\
\hline FEI FoV & instantaneous FoV covers an area on the ground surface of $36 \mathrm{~km} \times 36 \mathrm{~km}$ \\
\hline $\begin{array}{l}\text { FEI spatial sampling distance, along-track } \\
\text { and across-track }\end{array}$ & better than $0.6 \mathrm{~km}(\mathrm{G})$ and $0.75 \mathrm{~km}(\mathrm{~T})$ \\
\hline FEI measurement frequency & $\geq 5 /$ dwell time of the sounder \\
\hline FEI and FSI line of sight co-alignment & Co-aligned within 0.7 mrad \\
\hline FEI spectral channel & $11.5 \mu \mathrm{m}$ with $2.0 \mu \mathrm{m}$ width $(\mathrm{G}), 10.5 \mu \mathrm{m}$ with $1.5 \mu \mathrm{m}$ width $(\mathrm{T})$ \\
\hline FEI NEdT & smaller than $0.3 \mathrm{~K}(\mathrm{G}), 0.8 \mathrm{~K}(\mathrm{~T})$ at $210 \mathrm{~K}$ \\
\hline FEI ARA & better than $1 \mathrm{~K}(\mathrm{G})$ and $2 \mathrm{~K}(\mathrm{~T})$ at $210 \mathrm{~K}$ \\
\hline
\end{tabular}

and the output spectrum, but also to the L2 products, i.e. the difference between the true atmospheric state and the atmospheric components retrieved by the inversion module.

The usual way of performing L2 simulations consists in using the same code for simulating the instrumental spectrum, adding some random error to the exact values, and then retrieving the atmospheric state. In other words, errors aside, all the simulated cases could be exactly retrieved by the inversion code. In the case of the E2ES, it is possible to simulate external factors which must be definitively considered in the operative life of the instrument, and that can have an impact on the accuracy of the measurements. The main factors that can be simulated by the E2ES are:

- The instrument pointing errors;

- The lack of homogeneity in the instrument field of view;

- The errors due to the instrument hardware that are not fully represented by the Instrument Spectral Response Function (ISRF), which acts as a convolution kernel on the simulated high resolution radiances.

By adjusting the settings, the effect of each factor can be singled out. Unfortunately, due also to the ill conditioning of the retrieval problem, there is often no sensitivity in the measurements to address such fine details. Nevertheless, we are able to quantify the degradation of the results due to these external factors.

The operative L2 products are affected also by the model error. The radiative transfer is performed via a code that emulates the main mechanisms governing the diffusion of the radiance in the atmosphere. Our earth is however a much more complicated framework, the most evident difference is that the atmosphere is a continuous system, while a discretization technique has anyway to be applied in order to be fed into a computer. However, this lack of knowledge cannot be attributed to the instrument, so that adding a further uncertainty only interferes with the assessment of the instrument concept. Thus, the same forward 
model has been used in the generation of the scene and in the retrieval. This is equivalent to knowing perfectly how the radiative transfer works.

\section{E2ES architecture}

The E2ES is structured as a chain of modules, each performing a particular task. Each module relies on the data produced by the previous modules. The modules of the FORUM E2ES are:

- The Geometry Module (GM) calculates the true and error affected geographical coordinates of the field of view based on satellite location and pointing.

- The Scene Generator Module (SGM) calculates the high resolution radiances reaching the instrument, using the exact geographical coordinates and the prescribed atmospheric parameters. This is considered the true state.

- The Observing System Simulator (OSS) simulates the acquisition process of the instrument. Since in the preliminary phase, two different instrument concepts were available, two different configurations reproduce the two different hardwire instrument specifications.

- The Level 2 Module (L2M) uses the spectrum generated by the OSS and the noisy geographical coordinates to retrieve atmospheric parameters.

- The Performance Assessment Module (PAM) compares the true versus the retrieved L1 and L2 products and produces a report on the discrepancies.

The execution chain can be driven and checked from an external environment, the OpenSF structure, which is tailored for this kind of simulators. Figure 1 represents the E2ES structure with the data flow.

In the following subsections we briefly describe the purpose of the various modules and sketch the algorithms. A complete documentation may be found on the website devoted to FORUM at: https://www.forum-ee9.eu/.

\subsection{Geometry Module}

The Geometry Module (GM) is a C++ executable in charge of providing the geolocation and observations angles for every sample of FSI and FEI instruments. This requires computing the satellite orbit, the platform attitude, the instrument line of sight and the intersection of this line with the Earth for every epoch and instrument sample.

The GM produces two types of instrument geolocation grids annotated with the sampling time and the observation angles: error-free grids (on top of which the SGM simulates the TOA spectrum) and estimated grids (intended to feed the OSS with a geolocation that is affected by telemetry and calibration uncertainties). The GM output files are written in netCDF format.

The GM configuration file, which is based on XML syntax, allows the user defining several simulation parameters as the latitude and longitude of the region of interest, the acquisition start and stop times, the satellite orbit and attitude (optionally 
https://doi.org/10.5194/amt-2021-196

Preprint. Discussion started: 2 September 2021

(C) Author(s) 2021. CC BY 4.0 License.

(c) (i)
Atmospheric

Measurement

Techniques

Discussions

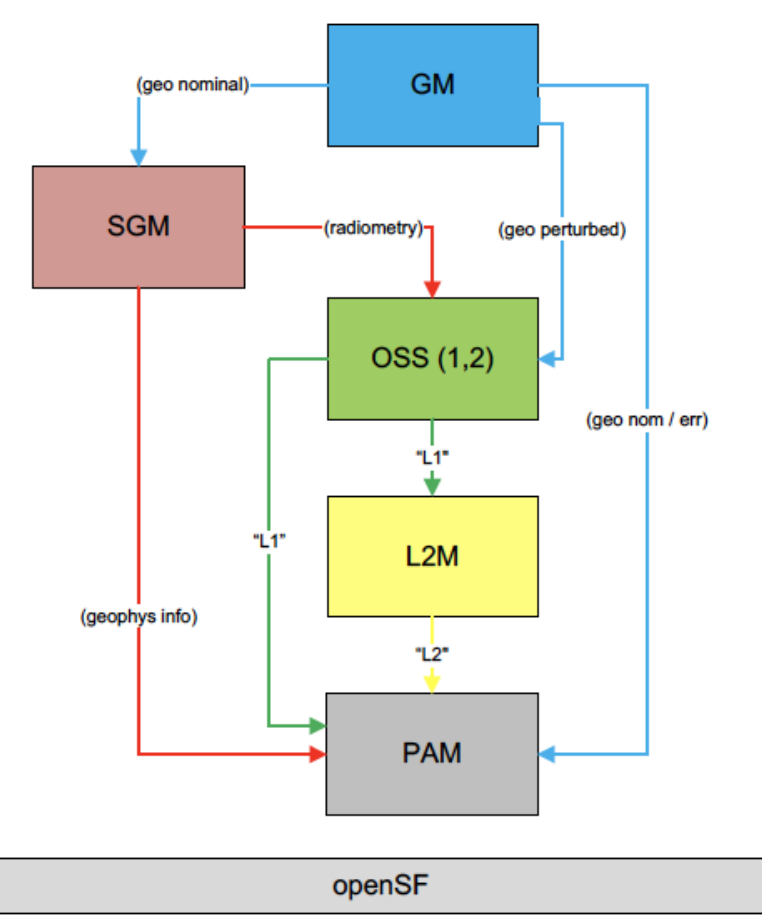

Figure 1. E2ES architecture and data flow.

also with knowledge errors), the IERS Bulletin providing Earth Rotation Parameters, the focal length, the mirror scanning law, the focal plane arrangement of FEI and FSI instruments and the spatial oversampling factor. The line of sight modelled with these parameters for every sample is then sent to the EO-CFI (Sánchez-Nogales, 2020) for computing the geodetic coordinates of its intersection with WGS84 geoid, or the selected digital elevation model.

\subsection{Scene Generator Module}

The Scene Generator Module (SGM) exploits the information on geolocation and observational geometry provided by the GM and performs a simulation of gridded spectral radiances to feed the FORUM observing system simulator. The SGM computes pixel by pixel radiances, with three different approaches: a fully automatic set up, a user driven scene definition, or a mixture of the two methods.

In the automatic approach, multiple databases are loaded to configure the surface, atmosphere, and cloud properties in accordance with geolocation, season, and time of the day without any intervention from the user. For storage limit, all the databases are currently available only for observations in the range $0-85^{\circ} \mathrm{N}$ and $0-30^{\circ} \mathrm{E}$, for two seasons (winter and summer), at four times of the day $(00,06,12,18 \mathrm{UTC})$. The surface properties are described by the spectral emissivity, obtained from the geolocated emissivity database by Huang et al. (2016), and the skin surface temperature, obtained from the ERA 5 reanalysis 
data (Hersbach et al., 2020). The atmospheric profiles of temperature, pressure, water vapor, and other 11 gases mixing ratios, interpolated at fixed altitude levels, are derived from the ERA 5 reanalysis and the IG2 climatological data (Remedios et al., 2007). The definition of the cloud geometrical and optical properties requires multiple steps. The ERA 5 reanalysis provides the total cloud cover (cloud fraction within the pixel) and, for each vertical layer, the cloud liquid water and/or ice content (LWC and IWC). It is assumed that liquid water and ice clouds can coexist in the same layer even if they are computationally treated as two separate components. The LWC and IWC values are used to establish if the scene is clear or cloudy (total cloud cover) and for the definition of the cloud vertical structure. For each layer of the model, the liquid water particles effective radii (Re) are computed from the LWC by using the parameterization described in Martin et al. (1994), whereas the ice particle radii are derived from the IWC and the application of the parameterization by Sun and Rikus (1999) in its revised version by Sun (2001). The cloud optical depth (OD, at $\left.900 \mathrm{~cm}^{-1}\right)$ is derived, layer by layer, from the information about LWC/IWC and the effective radii, by assuming optical properties for particle size distributions composed either of liquid water spheres (pre-computed) or of ice column aggregates crystals (Yang et al., 2013). Surface, atmosphere, and cloud properties are read and prepared as input for the LBLRTM (Clough et al., 2005), for clear sky description, and LBLDIS (Turner et al., 2003), for multiple scattering, cloudy sky description. These radiative transfer routines compute the spectral radiances for the FORUM imager and sounder. The default output spectral resolution is set to $10^{-2} \mathrm{~cm}^{-1}$ and $10^{-4} \mathrm{~cm}^{-1}$ for cloudy and clear sky simulations respectively.

In the user driven approach, the scene is manually configured and the user is able to set a large set of input parameters to customize the specific simulation. Basically, all the parameters defining the surface and cloud properties are user configurable. Nevertheless, the vertical atmospheric profiles are automatically selected from the ERA 5/IG2 databases for the required geolocation and data, as in the automatic approach. However, the SGM configuration parameters include the list of the atmospheric gases used for the simulation and thus, for each of them, their absorption properties can be turned off on request. The SGM allows the user to define surface temperature and emissivity, or to select specific emissivities from a list of 11 pre-defined surface types (e.g. water, desert, snow, deciduous vegetation). Two different kinds of surface can be assumed to exist in the FORUM field of view. The shape of the areas covered by different surfaces (with different emissivity and temperature) are defined by sectors or circles whose sizes are set by the user through proper geometric parameters in the SGM configuration list. The scene can be assumed as clear, overcast, or partially cloudy adding a third/fourth level of possible heterogeneity to the observed scene. In this last case, the cloud covers only a fractional area of the full FORUM field of view, corresponding to a sector or a circle with configurable geometric features such as in the case of the inhomogeneous surface scene. Liquid water, ice, or mixed phase clouds can be placed at different top altitudes and be of any thickness, even overlapping each other when two clouds are assumed in the same scene (e.g. liquid-ice or ice-ice). In case of ice clouds at the moment, the user is allowed to choose between two ice particle shapes (column aggregates or hexagonal plates) whose properties are derived from Yang et al. (2013), whereas the mixed phase particles are considered to be spheres with an ice core and a liquid coating. The cloud optical properties are defined by selecting the particle size distribution effective radius and the total optical depth (at $900 \mathrm{~cm}^{-1}$ ). All the configuration parameters are used to prepare the input files for the radiative transfer routines and to properly combine the output spectral radiances in the FORUM sounder representing the heterogenity according to the chosen spatial oversampling. 
Since the setting of the surface, atmospheric, and clouds properties are independent of each other, a mixed approach can be chosen by manually configuring some components and by letting the SGM configure the others.

Once the computations are performed for each pixel of the input grid (either over-sampled to the FSI footprint or not), the synthetic spectral radiances, the input configuration parameters, and other auxiliary information are passed to the observing system simulator and to the performances assessment module.

\subsection{Observing System Simulator}

Just as the real instrument, the Observing System Simulator (OSS) is composed by two parallel modules, simulating the embedded imager and the Fourier interferometer.

\subsubsection{FORUM Embedded Imager}

The FORUM Embedded Imager Observing System Simulator (FEI-OSS) is a compiled MATLAB executable in charge of simulating and calibrating the instrument acquisition of the FEI thermal infrared imager. The FEI is modelled as an uncooled bolometer with one spectral channel centred at $10.5 \mu \mathrm{m}$ and a 2-D sensor layout of typically 60 x 60 pixels (configurable).

The FEI OSS is composed of the instrument simulator (FEI-IS) and the L1b processor (FEI-L1).

The FEI-IS ingests the SGM output scene (possibly oversampled in the spatial domain), convolves the TOA spectra with the instrument Point Spread Function (PSF) in the spatial domain and integrates the resulting radiance with an Istrumental Spectral Response Function (ISRF) provided externally. Among its PSF modelling capabilities, the FEI-OSS is able to ingest an external PSF or to compute it by taking into account the configured parameters for the optics layout, the focal plane arrangement, the satellite motion blur and other high-frequency contributions as the AOCS jitter and the instrument micro-vibrations. Also, the channel centre is configurable.

The FEI-L1 translates the instrument output radiance into calibrated spectral radiance by taking into account noise and other instrumental errors. In particular, the noise is simulated in the brightness temperature domain with a Gaussian distribution of standard deviation equal to the configured NEdT. The calibrated radiance is finally written into the L1b product (netCDF file) along with the estimated geolocation provided by the GM module and some flags related to the FEI/FSI co-registration.

\subsubsection{FORUM Sounding instrument}

The FORUM Sounding Instrument Observing System Simulator (FSI-OSS) operates the software simulation of the performances of the FSI Fourier transform spectrometer from the starting point given by a set of input TOA spectral radiances provided by the Scene Generation Module (SGM). The simulator generates the interferograms corresponding to the outputs of the FSI instrument observing the selected scene, and then processes them with the level 1 data analysis code in order to recreate the observed TOA radiance.

In order to account for the effects of the finite FoV of the FSI instrument and telescope, the input radiances are provided on a sub-pixel matrix which covers the FSI footprint plus a suitable safety margin. Each of the sub-pixel is treated according 
to its specific observation geometry, specifically, its off-axis angle which produces a corresponding frequency shift due to the different pathlengths inside the FTS, and contributes accordingly to the output interferogram. This is the so-called selfapodisation effect.

Also, to correctly model the acquisition during the instrument dwell time, several inputs are provided to the OSS, corresponding to different times in the duration of the acquisition. Each input will have, in general, different line of sight parameters, and the result of the processing of each of the inputs is interpolated at interferogram level in order to produce the complete interferogram acquired in the duration of the dwell time.

The reference optical design used in the FSI-OSS consists in a Mach Zehnder interferometer, a design that provides the most general approach to a Fourier transform spectrometer. This design in fact allows us to place different sources on each of the two inputs of the interferometer, and different detectors on each of the two outputs. It also allows the use of separate divider and recombiner beamsplitters, which gives greater flexibility in the instrument design. Other optical schemes can be simulated just as particular cases of this more general design.

The main configuration parameters that allow to define the interferometer performances inside the FSI-OSS are the beam splitter reflection, transmission, and absorption coefficients, which are provided as complex, spectrally-dependent quantities to obtain the most accurate and general instrument response modeling (Bianchini et al., 2009, 2019).

One of the inputs of the interferometer is permanently set on a black-body source (reference black-body, RBB), while the other can be switched between the scene and two other reference sources (cold and hot black-bodies, CBB and HBB). This allows to operate the FSI-OSS in two different modes, scene and calibration, in order to simulate the full measurement process of the FSI instrument.

To obtain a meaningful representation of the interferogram that is observed on the outputs of the FSI the OSS needs to define a further set of transfer functions that describe the behaviour of the optical and electronic components of the system.

An overall, frequency dependent absorption coefficient can be defined in order to model possible effects due to optical components. Moreover, transfer functions describing the electronics response, expressed in terms of phase and amplitude are applied in order to correctly represent the output interferograms. The electronic response function can be further subdivided into detector response and preamplifier response, if needed. A last transfer function is used to model the effects of sampling and digitization, with the possibility of introducing arbitrary random or periodic sampling errors, or a calibration error in the interferogram time scale.

All the above described functions are coded in the instrument module, which outputs the simulated interferograms. The level 1 module has the function of transforming and calibrating the interferograms in order to reconstruct the observed scene radiance (Bianchini and Palchetti, 2008). The level 1 module is subdivided in three parts:

- Level 1a, which performs the Fourier transform of the interferogram, including the possible application of compensations for the different instrumental transfer functions, the zero path difference detection and the phase correction of the interferogram, producing as output the uncalibrated spectrum. 
- Level 1b, which performs the radiometric calibration of the uncalibrated spectrum and the estimation of the a-priori errors deriving from the calibration procedure (calibration error) and the detector noise (NESR error), providing as an output the measured atmospheric spectral radiance corresponding to the input scene as observed on each of the two instrument outputs.

- Level 1c, which performs the averaging of the two instrument outputs and the resampling of the average spectrum on a configurable frequency scale, in order to provide the level 2 module with a single measured spectrum with the required spectral sampling.

Last, a separate FSI-OSS module is used to calculate the ISRF corresponding to the configured FSI optical setup. This module performs the calculation of the ISRF taking into account the effect of the finite maximum optical path difference and the line broadening and shift due to the finite divergence of the radiation propagating inside of the interferometer (the self-apodization effect). The ISRF module generates an output file in a format which is directly compatible with the level 2 module.

\subsection{Level 2 module}

The L2M module is composed by the L2M_CIC (Cloud Identification and Classification) and L2M_I (Inversion) submodules. A python wrapper gathers the input data and runs the two submodules, providing the correct configuration files.

\subsubsection{Cloud Identification and Classification}

The main goal of the L2M_CIC submodule is to provide a classification of the observed spectrum. A machine learning algorithm named CIC (Maestri et al., 2019b) is embedded in the L2M_CIC routine and used to classify the FORUM sounder input spectrum. Four different pre-defined classes are used: clear sky, liquid water cloud, optically thick ice cloud, cirrus cloud. The classification is based on the comparison of the input spectrum with four training sets (TS), containing pre-computed spectra of the four classes. A principal component analysis determines the level of similarity of the input spectrum to the elements of each TS and selects the class of pertinence. If the spectrum is classified as cloudy (liquid water, thick ice cloud or cirrus cloud) key cloud parameters are derived and passed to the inversion module as first guess. The cloud parameters are: cloud top height, thickness, particle effective radius, and optical depth at $900 \mathrm{~cm}^{-1}$. Their values are derived from climatological data of cloud properties (Cloud CCI: Stengel et al. 2017; cirrus clouds: Veglio and Maestri 2011) and atmospheric profiles (ERA 5: Hersbach et al. 2020), according with the cloud classification and with the geolocation, season, and observed brightness temperature at $900 \mathrm{~cm}^{-1}$.

The CIC algorithm classification is based only on FORUM sounder spectral radiance and thus regards the radiance from an extended (about $15 \mathrm{~km}$ diameter) field of view. The classification is provided independently of the presence or not of sub-pixel heterogeneities. Therefore, a scene classified as cloudy could be the results of a field of view that is only partially covered by clouds plus a clear sky fractional area, and vice versa. The L2M_CIC exploits the FORUM imager data to pair the spectrum classification with a scene homogeneity information. The imager pixels radiances are first converted into brightness 
temperatures (BT), then, a BT distribution (histogram) is analysed with a custom made fitting function. A simple algorithm identifies the BT distribution modes and splits the imager pixels into homogeneous groups of pixels characterized by limited BT variation. Thus, the L2M_CIC submodule provides auxiliary information concerning the number of homogeneous areas identified at FORUM sounder sub-pixel level, their average BT and standard deviation. This is used as a quality flag for the inversion results, since non-homogeneous scenes are in any case managed as homogeneous in the retrieval process. A land mask for the imager field of view is also defined, based on the geo-referred database GSHHG (Wessel and Smith, 1996, and updates), so that BT inhomogeneities caused by different sea-land surfaces are easily identified, especially in clear sky.

\subsubsection{Inversion}

The purpose of the inversion module is to solve the inverse radiative transfer problem, retrieving any combination of surface temperature, surface emissivity and vertical profiles of temperature and water vapour in clear sky conditions, and cloud parameters in cloudy sky conditions. The retrieval algorithm is based on the classical OE (Optimal Estimation) method (Rodgers, 2000). The inversion is performed via the Gauss-Newton sequence, with the Marquardt modification to ease Hessian inversion and to compensate for the non-linearity of the forward model. The a-posteriori IVS (Iterative Variable Strength) regularization technique (Ridolfi and Sgheri, 2011; Eremenko et al., 2019; Sgheri et al., 2020) is then applied to smooth out the retrieved profiles. In accordance with the SGM, the code makes use of the LBLRTM forward model in clear sky conditions, and the LBLDIS frontend to the DISORT multiple scattering code in cloudy sky conditions. We used an accelerated version of DISORT (Sgheri and Castelli, 2018), able to reduce the multiple scattering computation time. The instrumental effects are factored via the convolution with the ISRF function. In this early version of the FORUM E2ES the decision was made to maintain a frequency dependent ISRF. Consequently, the convolution cannot be computed in the Fourier domain. We obtained the actual ISRF on a rather fine grid with sampling step of $3 \mathrm{~cm}^{-1}$. However, using the apodized sampled ISRF can increase the chi-square by up to $30 \%$ with respect to the unapodized spectrum, due to the fact that we exchange the interpolation and apodization operators. The correct sequence of first convolving with the sampled ISRF and then convolving with the apodization kernel increases the computation time and does not remove the error introduced by cutting the tails of the ISRF, which is one of the purposes of apodization. Thus, at least in this stage, we preferred to use the unapodized spectrum.

\subsection{Performance Assessment Module}

The Performance Assessment Module (PAM) is a MATLAB executable aimed to compute and plot the retrieval accuracy of L2M. In particular this module also allows inspecting the SGM output (TOA spectral radiance) and plotting the L2M vs. SGM profiles including error bars and residual statistics for clear-sky retrievals (emissivity, skin temperature, atmospheric temperature, atmospheric water vapor and precipitable water vapor) and cloudy-sky retrievals (cloud top height, cloud optical depth, cloud particle size, cloud thickness and ice/liquid water path). The PAM is also able to plot the Averaging Kernel (AK) profiles computed by the L2M and the FWHM vertical resolution (averaging kernels may be optionally convolved with the SGM reference for comparison to the L2M retrievals). 
Table 4. List of homogeneous scenes considered

\begin{tabular}{|c|l|l|l|l|l|}
\hline Case \# & Scene & Geolocation & Surface & Time & Type of cloud adopted in cloudy case \\
\hline 1.1 & Tropical & Sahara desert: $24.75^{\circ} \mathrm{N}-24.75^{\circ} \mathrm{E}$ & Desert & Summer & Cirrus on desert \\
\hline 2.1 & MidLat 2 & Mediterranean: $33.75^{\circ} \mathrm{N}-18.75^{\circ} \mathrm{E}$ & Water & Summer & Cirrus on ocean \\
\hline 3.1 & MidLat 1 & Mediterranean: $39.75^{\circ} \mathrm{N}-6.75^{\circ} \mathrm{E}$ & Water & Summer & Marine stratus \\
\hline 4.1 & MidLat 3 & Black forest: $50.25^{\circ} \mathrm{N}-6.75^{\circ} \mathrm{E}$ & Deciduous & Winter & Continental stratocumulus \\
\hline 5.1 & Polar & Finland: $68.25^{\circ} \mathrm{N}-18.75^{\circ} \mathrm{E}$ & Fine snow & Winter & Ice cloud on snow ground \\
\hline 6.1 & MidLat 4 & Po Valley: $45^{\circ} \mathrm{N}, 11.25^{\circ} \mathrm{E}$ & Deciduous & Summer & Cumulus nimbus \\
\hline 7.1 & Polar & Kuusiluoto-Finland: $65.75^{\circ} \mathrm{N}-24.25^{\circ} \mathrm{E}$ & Ice & Winter & - \\
\hline
\end{tabular}

\section{Test scenarios}

The FORUM E2ES is meant to study the potentialities and criticalities of the FORUM mission for realistic characteristics of the instrument and for different measurement scenarios.

The different measurement scenarios, used both for the validation of the different modules and the assessment tests, were defined in the homogeneous case to be representative of seasons and latitudes in clear and cloudy conditions.

The FEES is able to simulate FORUM measurements all over the globe, provided the climatological data is included in the SGM dataset (see the relevant section).

Within the area selected for the FEES study, seven scenarios were identified: three extreme cases (two in polar regions in winter with two different surface characteristics, ice and fine snow, one in the tropics on the desert) and four cases at Middle Latitude (two on the sea, in summer, two on vegetation, one in summer and one in winter). These scenes can be simulated either in clear-sky or in cloudy-sky.

Table 4 provides, for each scene, geolocation, type of surface, and time, as well as the type of cloud assumed for each scene in the case of cloudy sky. The cloudy sky scenarios are computed adding to the clear sky atmosphere different homogeneous types of clouds filling all the FoV (see Table5).

355 While with this approximation we depart from the real data, it does not have an impact on the assessment of the performances of the FEES.

In the assessment tests, we also used the capability of SGM to simulate heterogeneous scenes, assuming a FoV covered by portions of different homogeneous atmospheres. 
Table 5. Cloud characteristics associated to scenes considered

\begin{tabular}{|c|l|c|r|r|r|r|}
\hline Case \# & Cloud Type & Type & Top $[\mathrm{km}]$ & Thickness $[\mathrm{km}]$ & Optical Depth & Radius $[\mu \mathrm{m}]$ \\
\hline 1.2 & Cirrus on desert & ice & 12 & 2 & 1 & 10 \\
\hline 2.2 & Cirrus on ocean & ice & 15 & 1 & 0.3 & 6 \\
\hline 3.2 & Marine stratus & water & 1.25 & 0.5 & 20 & 10 \\
\hline 4.2 & Continental stratocumulus & ice & 10 & 2 & 6 & 30 \\
\hline 5.2 & Ice cloud on snow ground & ice & 8 & 6 & 300 & 18 \\
\hline 6.2 & Cumulus nimbus scene & ice & 11 & 2 & 80 \\
\hline
\end{tabular}

\section{E2ES validation and assessment tests}

Assessment tests were performed to evaluate the quality of the L2 products, assuming the FORUM instruments in a configuration satisfying the L1 goal requirements, for the different scenarios identified to cover a variety of atmospheric characteristics. These tests were performed for both homogeneous and inhomogeneous scenes, in both clear sky and cloudy sky.

The results obtained in the homogeneous case represent the best that can be obtained from FORUM for the different scenes, and hence the quality of the products obtained in these conditions constitutes the reference for the other tests, performed with heterogeneous scenes.

The L2 clear sky products of the E2E simulator were also validated by comparing them with the outputs obtained, starting from the same observations generated by the FSI module, by an already validated correlative code. The reference code used in clear sky is KLIMA (Kyoto protocoL Informed Management of the Adaptation).

Conversely the L2 cloudy sky products of the E2E simulator were compared with the outputs of the reference SACR (Simultaneous Atmospheric and Cloud Retrieval) code. However, due to some differences in the model and approach, we cannot speak rigorously of a validation.

\subsection{Homogeneouos Clear sky cases}

The seven clear sky test case listed in Table 4 were analyzed. The simulated observed spectra were generated by the E2ES chain, including the GM, the SGM, the FEI and the FSI. The reference atmospheric state was constructed using ECMWF information. The vertical grid used to represent the atmospheric profiles in both the SGM and the L2M is composed of levels distant about $0.5 \mathrm{~km}$ up to $15 \mathrm{~km}, 1 \mathrm{~km}$ from 15 to $25 \mathrm{~km}, 5 \mathrm{~km}$ from 25 to $40 \mathrm{~km}, 10 \mathrm{~km}$ up to $80 \mathrm{~km}$. The used noise error and spectral resolution are aligned with information provided by the industrial consortia, compliant with the goal parameters specified in Tables 1 and 3. Temperature, Water vapour, surface temperature and surface emissivity are retrieved simultaneously by L2M using the Optimal Estimation approach. The Covariance Matrices of the a-priori vertical profiles of temperature and water vapour are built assuming the errors defined by the UK MetOffice for routine assimilation of IASI products into their operational Numerical Weather Prediction (NWP) system (see Figure 5). Indeed, IASI-NG measurements are planned to be 
used in synergy with the FORUM measurements. For the emissivity, which is represented on a $5 \mathrm{~cm}^{-1}$ spaced grid, we used a constant error of 0.05 , with a correlation length in wavenumber of 50. For surface temperature an a-priori error of $2 \mathrm{~K}$ was used. The a-priori profiles used for these tests for all the retrieved variables are given by the truth perturbed with one standard deviation of the a-priori error. This is the most sensible choice, especially for emissivity. In fact, the sensitivity to the emissivity in the spectrum is different in different regions of the spectrum. If a stochastic perturbation were used, the results would depend on how large is the actual perturbation in the frequency ranges where there is sensitivity. For the initial condition of the retrieval we used for temperature, water vapour and surface temperature the values taken from the climatology, obtained as the 10-years local average in the month of ECMWF analysis profiles, while for emissivity the a-priori values were used. The reason for this difference is that the GN method only looks for local minima. Starting from the a-priori, the chance to obtain a solution close to the a-priori itself is not negligible. On the other hand, the dependence of the spectrum from emissivity is linear, so that the possibility of finding a local minimum is minimal.

\subsubsection{Validation with KLIMA code}

The KLIMA code, used to validate the L2M module in clear-sky conditions, performs the retrieval of the atmospheric profiles and surface parameters from the spectral radiance measurements. The code consists of two distinct modules, the Forward Model (FM) and the Retrieval Model (RM). The RM has been developed in the context of the KLIMA ESA study (Cortesi et al., 2014) by upgrading the algorithm employed for the analysis of REFIR-PAD measurements (Bianchini et al., 2008) adapted in turn from the MARC inversion code for the MARSCHALS ESA study (Carli et al., 2007). The FM is a line-byline radiative transfer model, with capability to simulate wideband spectral radiances, based on the following key features: radiative transfer calculations performed using Curtis-Godson approximation; atmospheric line shapes modelled with Voigt profile and atmospheric continuum model takes into account the main contributions from $\mathrm{N}_{2}, \mathrm{O}_{2}, \mathrm{O}_{3}, \mathrm{H}_{2} \mathrm{O}$, and $\mathrm{CO}_{2}$. The spectroscopic database adopted for the simulations are AER version aer_v_3.7 (http://rtweb.aer.com/line_param_frame.html), the atmospheric continuum is modelled using the routine MT_CKD_3.3 (http://rtweb.aer.com/continuum_whats_new.html) considering the contribution of the lines external to the region of $\pm 25 \mathrm{~cm}^{-1}$ from the line center. A dedicated spectroscopic database and line shape are adopted for $\mathrm{CO}_{2}$, to take into account the line-mixing effect when using the AER (Atmospheric and Enviromental Research) spectroscopic database (http://rtweb.aer.com/line_param_frame.html). Moreover, the correction of the Planck function (Clough et al., 1992) is included to take into account the optical depth of the atmospheric layer at the different frequency. The validation of KLIMA FM was conducted in the context of IASI data analysis (Cortesi et al., 2014) by comparing synthetic IASI measurements generated by the KLIMA FM code with those of the FM of the LBLRTM. The RM uses a constrained NLSF approach and the cost function to be minimized taking into account the a priori information (optimal estimation method) and the Marquardt parameter. The code implements the multi-target retrieval: more than one species is simultaneously retrieved along with many other atmospheric, surface, and instrumental parameters. A complete Covariance Matrix (CM) can be used, including both the measurement errors and the errors in the calibration procedure and/or in the estimation of the FM parameters. The purpose of the validation was to compare the retrieved quantities and the corresponding errors (given by the mapping of measurement error on retrieved quantities) provided by the L2M of the E2E simulator and by 
Table 6. KLIMA/L2M_I retrieval quality comparison

\begin{tabular}{|c|c|c|c|}
\hline Case \# & Initial reduced $\chi^{2}$ & Gauss iterations & Final reduced $\chi^{2}$ \\
\hline 1.1 & $20.46 / 20.45$ & $3 / 5$ & $1.07 / 1.09$ \\
\hline 2.1 & $2.86 / 2.86$ & $4 / 3$ & $1.09 / 1.09$ \\
\hline 3.1 & $4.20 / 4.20$ & $4 / 3$ & $1.08 / 1.08$ \\
\hline 4.1 & $2.41 / 2.40$ & $3 / 3$ & $1.07 / 1.08$ \\
\hline 5.1 & $3.66 / 3.65$ & $3 / 3$ & $1.07 / 1.08$ \\
\hline 6.1 & $3.54 / 3.54$ & $3 / 3$ & $1.08 / 1.08$ \\
\hline 7.1 & $7.19 / 7.19$ & $9 / 4$ & $1.10 / 1.10$ \\
\hline
\end{tabular}

Table 7. KLIMA/L2M_I Surface Temperature Retrieval Comparison

\begin{tabular}{|l|r|r|r|r|r|r|r|}
\hline Case \# & $\mathbf{1 . 1}$ & $\mathbf{2 . 1}$ & $\mathbf{3 . 1}$ & $\mathbf{4 . 1}$ & $\mathbf{5 . 1}$ & $\mathbf{6 . 1}$ & $\mathbf{7 . 1}$ \\
\hline KLIMA-L2M_I [K] & 0.25 & -0.10 & 0.02 & 0.19 & -0.01 & -0.05 & 0.03 \\
\hline KLIMA error [K] & 0.36 & 0.33 & 0.31 & 0.30 & 0.21 & 0.34 & 0.28 \\
\hline L2M_I error [K] & 0.34 & 0.34 & 0.31 & 0.30 & 0.21 & 0.34 & 0.27 \\
\hline
\end{tabular}

KLIMA when starting from the same conditions. From the statistical point of view the validation may be considered as reached if the differences in the estimated retrieval error are negligible and differences between the retrieved values are smaller than the retrieval error. However, we also set a goal at $10 \%$ of the retrieval error, and we checked that, on average, the differences lie within that bound.

In Table 6 we report the comparison between the initial and final reduced $\chi^{2}$ obtained using KLIMA end L2M_I. The number of Gauss iterations is also reported. Each row of the table refers to a different scenario. The Reduced $\chi^{2}$ differences (both initial and final) are smaller than 0.02. Although the convergence criteria are the same (i.e. reduction of the $\chi^{2}$ less than $1 \%$ ), the number of Gauss iterations in some cases is different. This is due to differences on reduced $\chi^{2}$ of the order of 0.001 .

In Figure 2 we report the comparison between the retrieved $\mathrm{H}_{2} \mathrm{O}$ value profile and error profile obtained using KLIMA and L2M_I. The differences are divided by the mean retrieval error. The differences between retrieval errors are smaller than 5\% and differences between profiles retrieved by the two codes are smaller than 0.4 times the retrieval error, with the maximal difference concentrated in the lower tropospheric region.

Results for temperature validation are reported in Figure 3. The differences between retrieval errors are smaller than $25 \%$ and the differences between profiles retrieved by the two codes are mostly smaller than 0.5 times the retrieval error. 

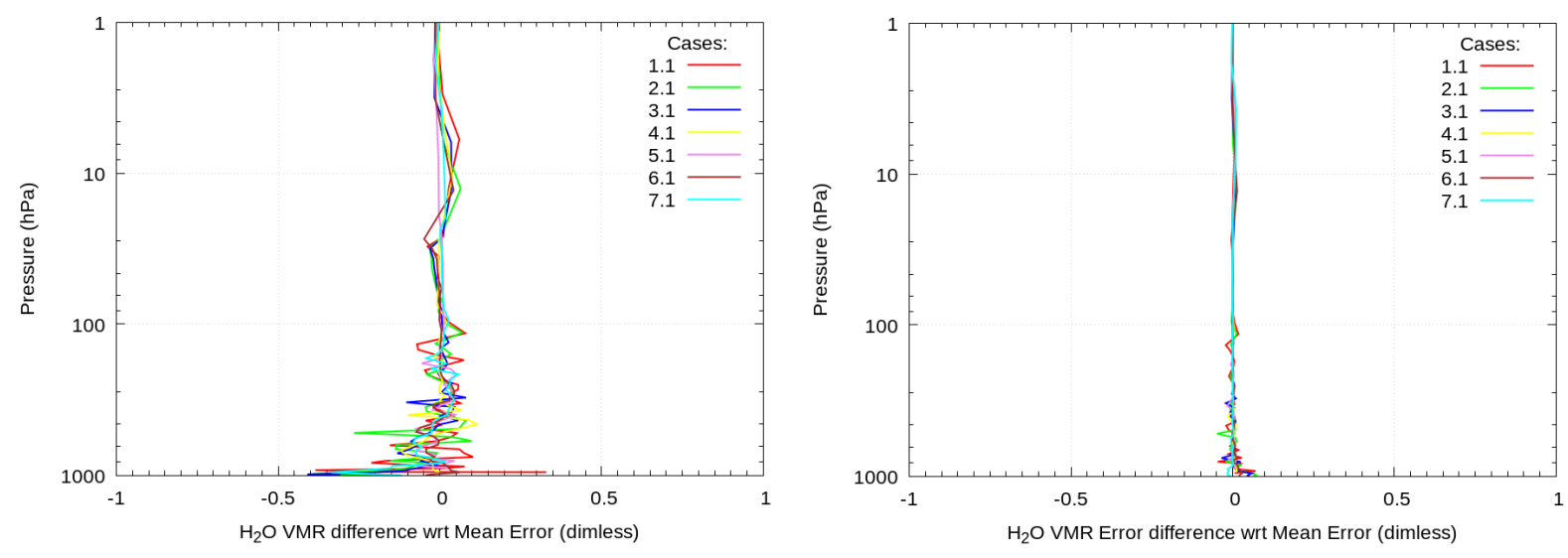

Figure 2. Left plot: Difference of the water vapour profile obtained from KLIMA and L2M_I divided the mean value of the retrieval error for the seven analysed scenarios. Right plot: Difference of the water vapour retrieval error profile obtained from KLIMA and L2M_I divided the mean value of the retrieval error for the seven analysed scenarios.
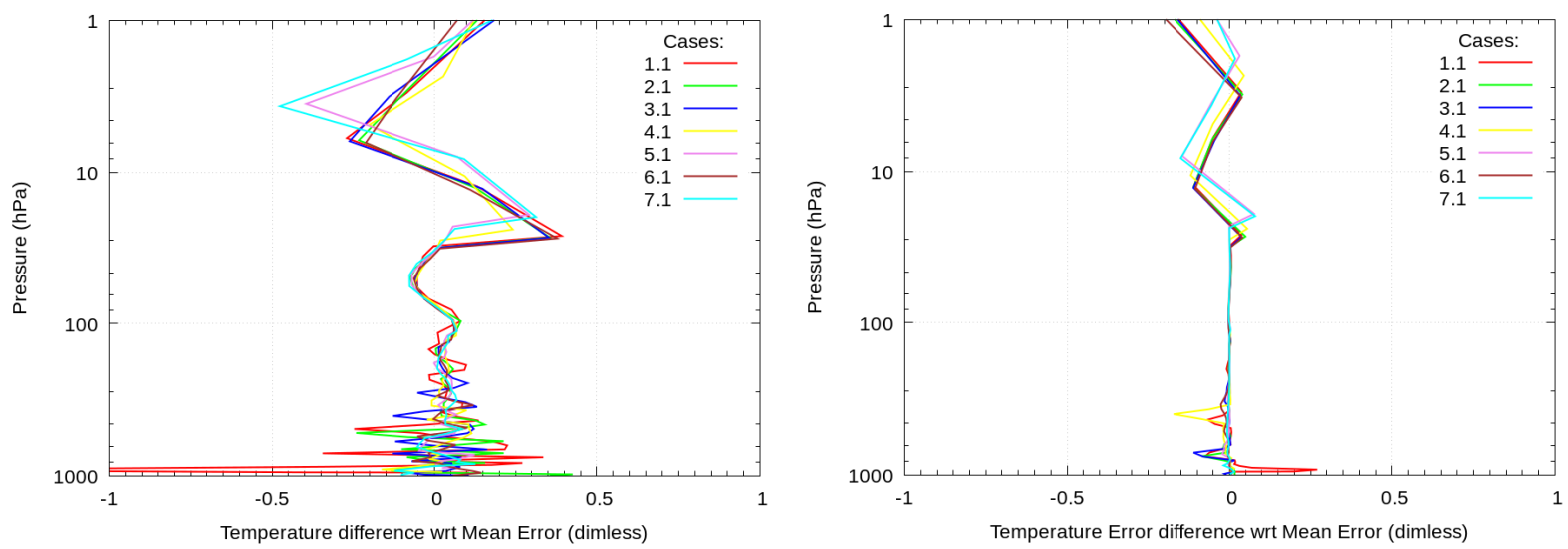

Figure 3. Left plot: Difference of the temperature profile obtained from KLIMA and L2M_I divided the mean value of the retrieval error for the seven analysed scenarios. Right plot: Difference of the temperature retrieval error profile obtained from KLIMA and L2M_I divided the mean value of the retrieval error for the seven analysed scenarios. 

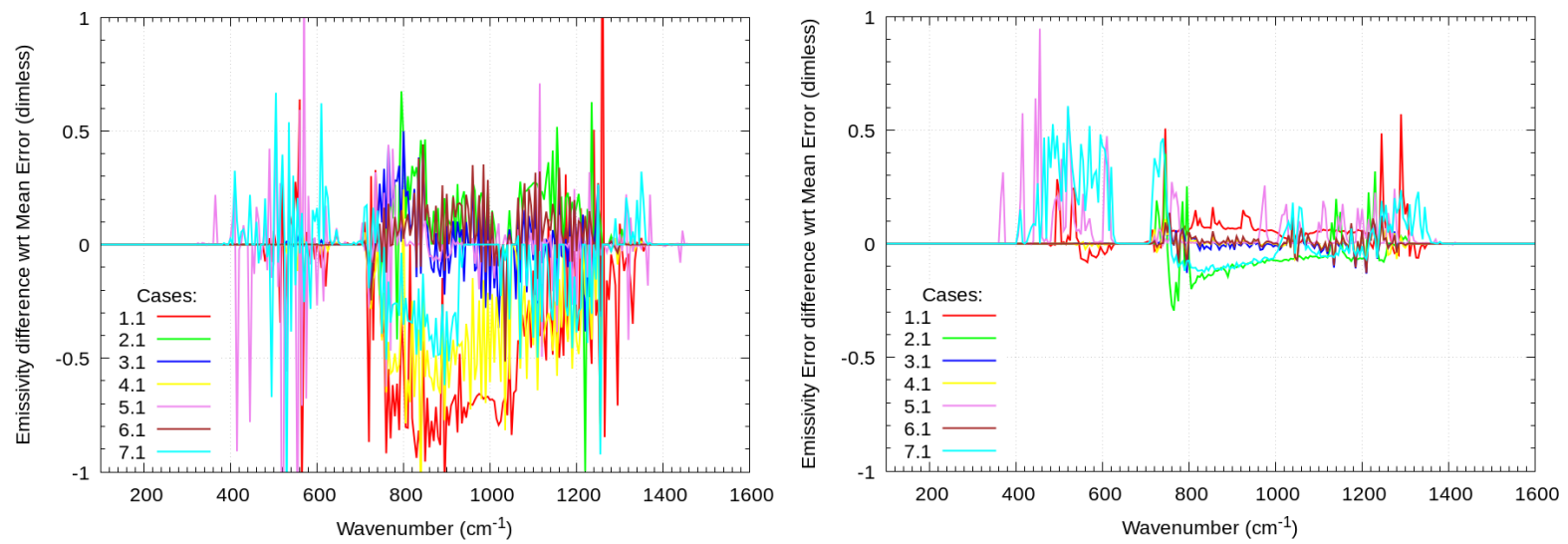

Figure 4. Left plot: Difference of the surface emissivity spectrum obtained from KLIMA and L2M_I divided the mean value of the retrieval error for the seven analysed scenarios. Right plot: Difference of the surface emissivity retrieval error spectrum obtained from KLIMA and L2M_I divided the mean value of the retrieval error for the seven analysed scenarios.

In Table 7 we report the comparison between the retrieved surface temperature and error obtained using KLIMA and L2M_I. Each row of the table refers to a different scenario. The differences between the retrieval errors are smaller than $15 \%$ and the differences between the profiles retrieved by the two codes are mostly smaller than 0.3 times the retrieval error.

In Figure 4 we report the comparison between the spectrum of the retrieved surface emissivity and its error spectrum obtained using KLIMA and L2M_I. The differences are divided by the mean retrieval error. The differences between retrieval errors are smaller than $30 \%$ and the differences between profiles retrieved by the two codes are mostly smaller than 0.5 times the retrieval error. The larger differences in the MIR range that show up in some cases are mainly due to the high negative correlation between surface temperature and emissivity. For the retrieval procedure, the effect of a lower surface temperature with a higher emissivity is similar to that of a higher surface temperature with a lower emissivity.

\subsubsection{Discussion of the results}

We describe here a selection of the results. In Figure 5 we report the retrieved profiles, the retrieval errors (estimated from the diagonal elements of the $\mathrm{CM}$ ) and the differences between the retrieved and the true profiles of temperature and water vapour for all the seven cases.

For both temperature and water vapour the difference between the retrieved profile and the truth is well within the retrieval error for most of the points and all cases. Despite the variability of the temperature and water vapour profiles in the various cases, the sensibility of the temperature and water vapour retrieval, given by the retrieval error, does not change significantly and the requirements on precision are met in all cases. The retrieval reduces the a priori uncertainty by a factor 2 for temperature 

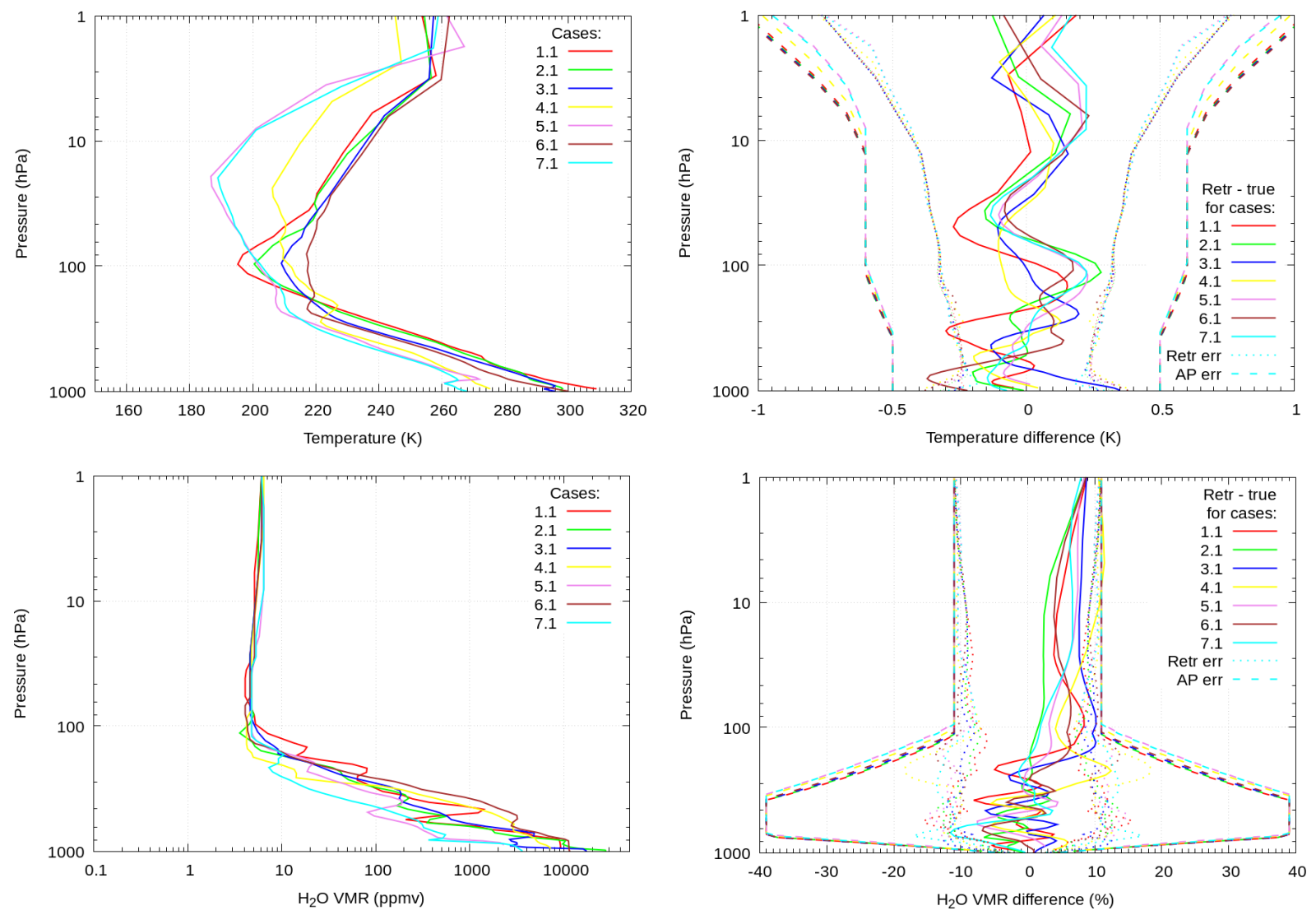

Figure 5. Retrieved temperature profiles for the seven clear sky cases (top left). Difference between retrieved and true temperature profiles, with retrieval and a-priori error (top right). Retrieved water vapor profiles for the seven clear sky cases (bottom left). Difference between retrieved and true water vapour profiles, with retrieval and a-priori error (bottom right).

and a factor 5 for water vapour. In Figure 6 we report the typical behaviour of the AKs for temperature and water vapour. Values of the diagonal elements significantly smaller than 1 in the troposphere does not depend only on the fact that the used a-priori errors are small and hence the information gain is limited, but also on the very fine used retrieval grid in the troposphere which determines small information content in each retrieved component. A most useful information in this case is provided by the number of degrees of freedom (DOFs), given by the trace of the averaging kernel matrix. The DOFs of the temperature profile varies from 7 for case 1.1 (tropical dry case) to about 6 for case 2.1 (middle latitude case) to 5 for case5.1 (polar case), with $75 \%$ of the DOFs being concentrated in the lowest $25 \mathrm{~km}$. For water vapour profile the number of DOFs varies from 8.2 for case 1.1 to 7 for case 2.1 to 5.4 for case 5.1, with more than $90 \%$ of DOFs being concentrated in the lowest $25 \mathrm{~km}$. Table 8 reports the results of surface temperature retrieval. While the retrieval error is smaller by at least a factor of 5 than a-priori uncertainty, all cases show a good performance (differences between retrieved and true value smaller than the retrieval error). The exception is case 1.1 (desert at noon in summer), where there is a negative bias of 0.8 degrees, which is larger than the 

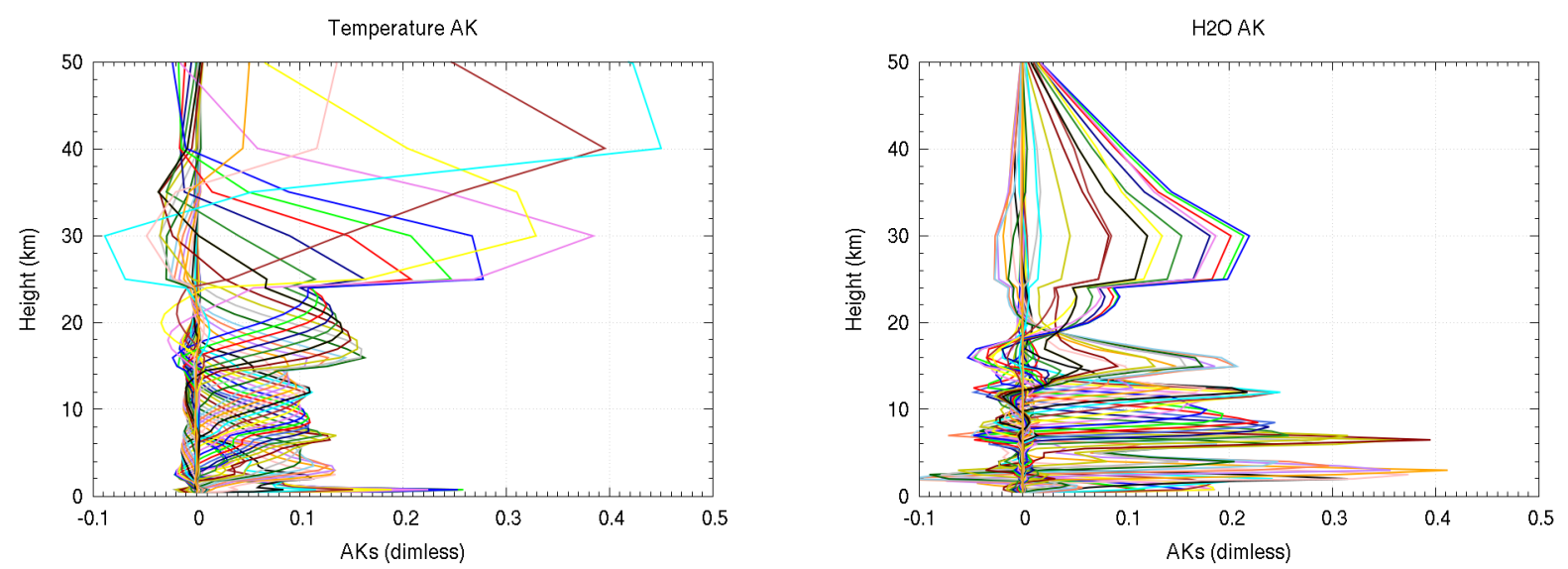

Figure 6. Averaging kernels for temperature (left panel) and water vapour (right panel) for case 1.1 (clear sky desert at noon in summer).

Table 8. Results for the retrieval of surface temperature for the seven clear sky cases.

\begin{tabular}{|c|r|r|r|r|r|}
\hline Case \# & Retrieved-True & Retrieval error & A-priori-True & A-priori error & Initial Guess-True \\
\hline 1.1 & -0.815 & \pm 0.34 & 2 & \pm 2 & -1.333 \\
\hline 2.1 & -0.34 & \pm 0.3 & 2 & \pm 2 & -0.99 \\
\hline 3.1 & 0.091 & \pm 0.24 & 2.1 & \pm 2 & -0.073 \\
\hline 4.1 & 0.098 & \pm 0.279 & -0.65 & \pm 2 & -0.436 \\
\hline 5.1 & 0.14 & \pm 0.21 & 2 & \pm 2 & 2.34 \\
\hline 6.1 & -0.224 & \pm 0.393 & -1.775 & \pm 2 & 1.468 \\
\hline 7.1 & -0.073 & \pm 0.23 & 2 & \pm 2 & -4.9 \\
\hline
\end{tabular}

retrieval error. This is due to a large negative correlation between surface temperature and emissivity in the MIR. This is further discussed when dealing with the results for surface emissivity.

For emissivity retrieval, we can divide our sample into three groups, with similar atmospheric conditions:

- The polar cases (5.1 and 7.1), high latitude and dry atmosphere (snow and ice).

- The middle latitude cases $(2.1,3.1,4.1,6.1)$, water vapour rich atmosphere over water or deciduous ground.

- The desert case (1.1), which combines a dry atmosphere and hot surface temperature. Also, the emissivity pattern has substantial features in the MIR region. 

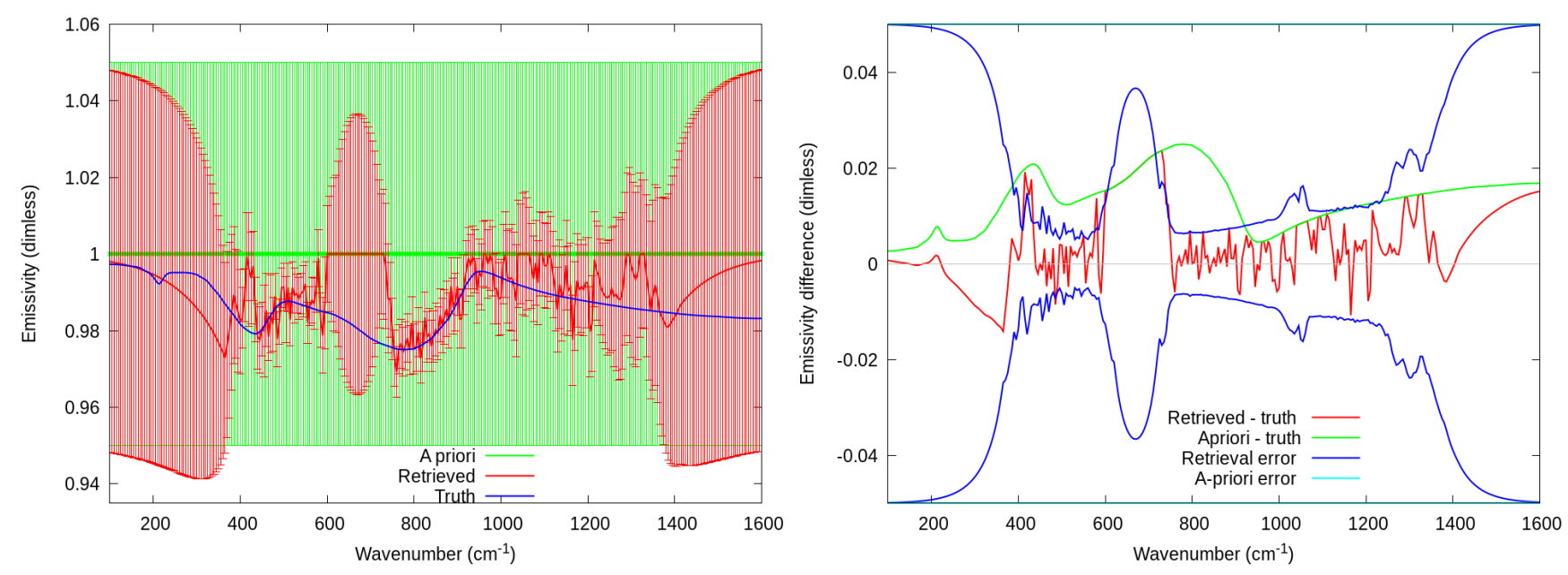

Figure 7. Emissivity retrieval for case 5.1 (polar case on fine snow). Left panel: a-priori profile with error bars (green), retrieved profile with error bars (red), truth (blue). Right panel: difference between retrieved and true (red), retrieval error (blue), difference between a-priori and model (green), a-priori error (cyan).

For the polar group we show case 5.1 in Figure 7 . We note that, as expected due to the dry atmosphere which makes it transparent to the surface also in the FIR, there is sensitivity to the measurements both in the FIR and in the MIR regions.

This emerges also from the analysis of the AKs of the frequency dependent emissivity profile (see Figure 8), characterized by values of the diagonal matrices very close to 1 in the $500-600 \mathrm{~cm}^{-1}$ and in the $700-1000 \mathrm{~cm}^{-1}$ regions. In this regions the apriori information contributes only very marginally to the inversion and the grid is sufficiently coarse to allow each component of the used frequency grid to capture significant information. The number of DOFs for the surface emissivity is 105 for the full spectral range, with the FIR spectral region contributing with 30 DOFs.

For the middle latitude group we show case 2.1 in Figure 9. In these cases, the rich water content in the troposphere masks the emissivity signal in the FIR, so there is only sensitivity in the MIR atmospheric window, where the transparency of the atmosphere allows to get information on the surface. This is confirmed by the contribution to the total number of DOFs for the surface emissivity retrieval. No contribution to the DOFs comes from the FIR region, while the total number is 74 .

Finally in case 1.1 the retrieval of the emissivity shows some sensitivity in the FIR region (about 5 DOFs from the FIR) on a total of 109 from the full spectral region), but also a positive bias of about 0.01 in the MIR region, as shown in Figure 10.

With sensitivity tests we discovered that the bias does not depend on the particular choice of the spectrum noise. Also, the bias shows up also when retrieving only emissivity and skin temperature. The sign of the bias depends on the sign of the perturbation of the a-priori emissivity, while the initial guess of the emissivity and the initial guess and a-priori of the surface temperature have no effect. The effect is due to a strong anticorrelation between emissivity and surface temperature, which reaches -0.9 in the MIR region. There are different ways of solving this problem and ad-hoc studies to optimize the retrieval 


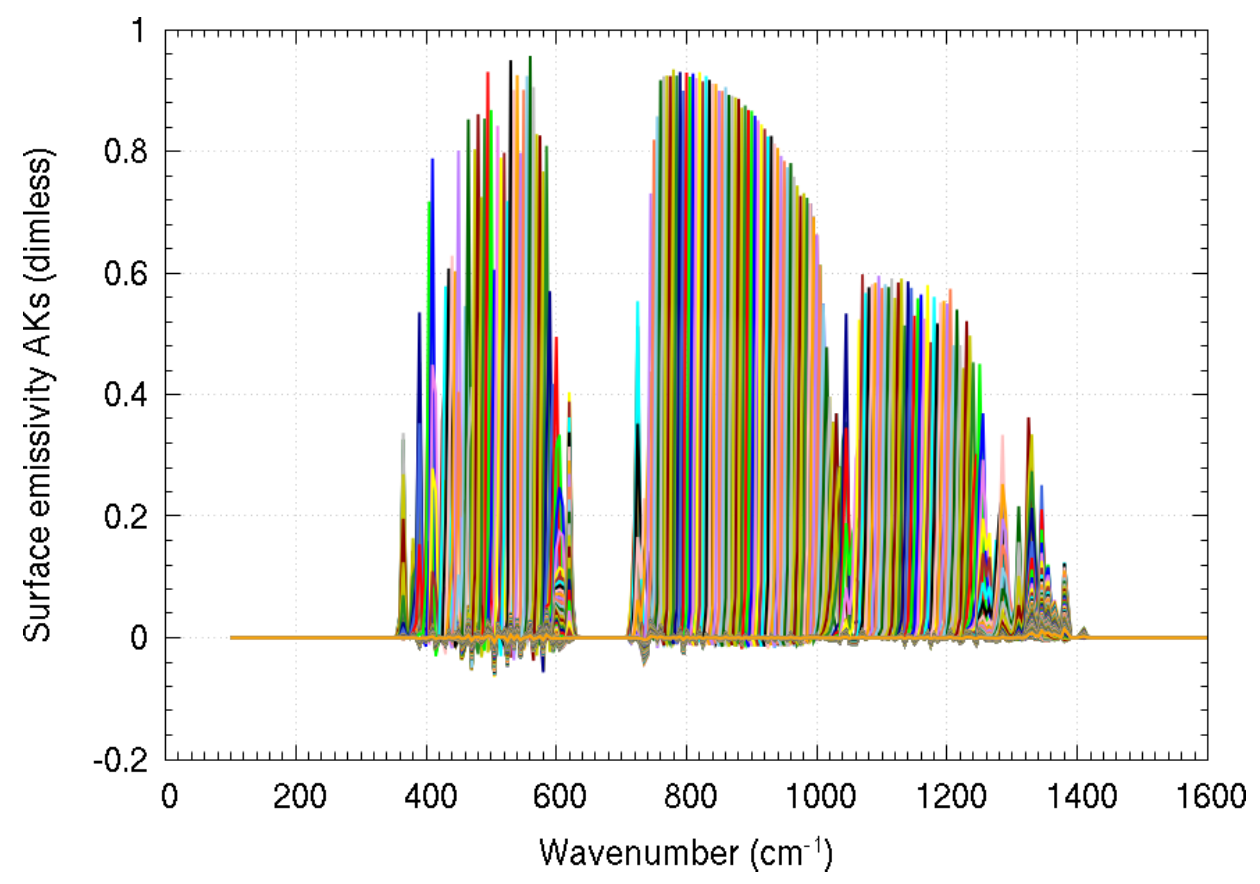

Figure 8. Averaging Kernels of the frequency dependent emissivity profile for case 5.1 (polar case on fine snow).
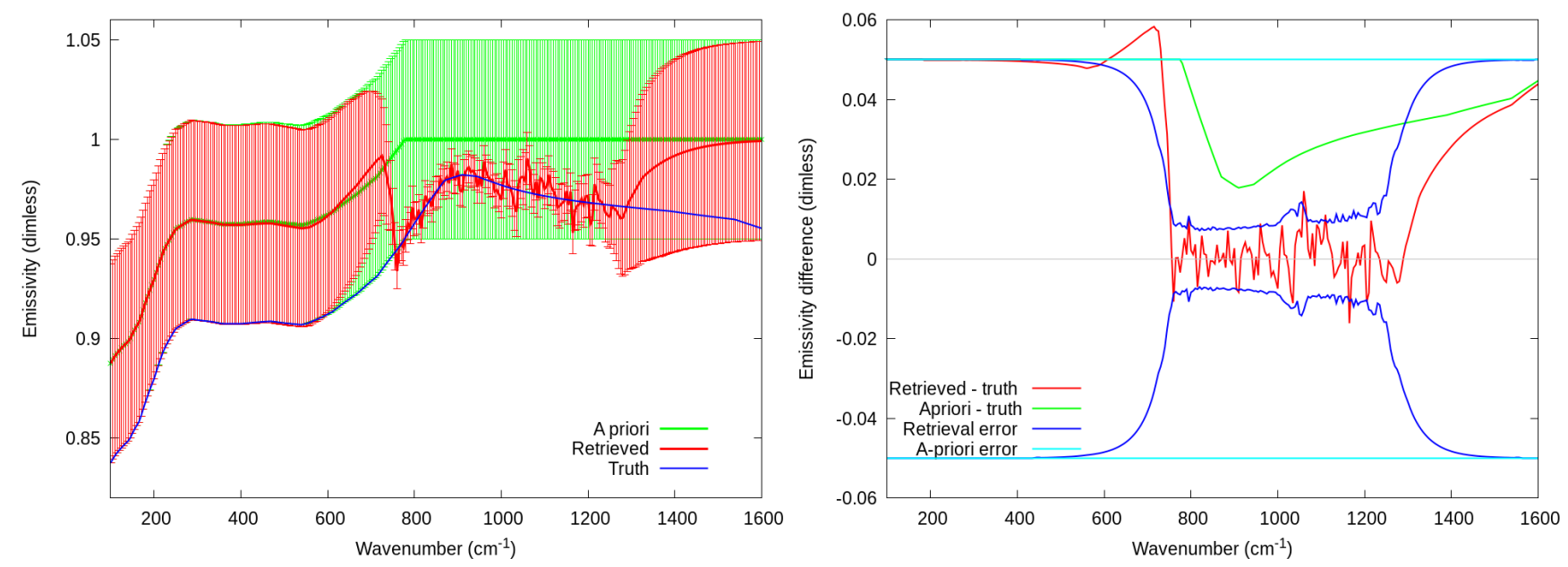

Figure 9. Emissivity retrieval for case 2.1 (middle latitude on water). Left panel: a-priori profile with error bars (green), retrieved profile with error bars (red), truth (blue). Right panel: difference between retrieved and true (red), retrieval error (blue), difference between a-priori and model (green), a-priori error (cyan). 

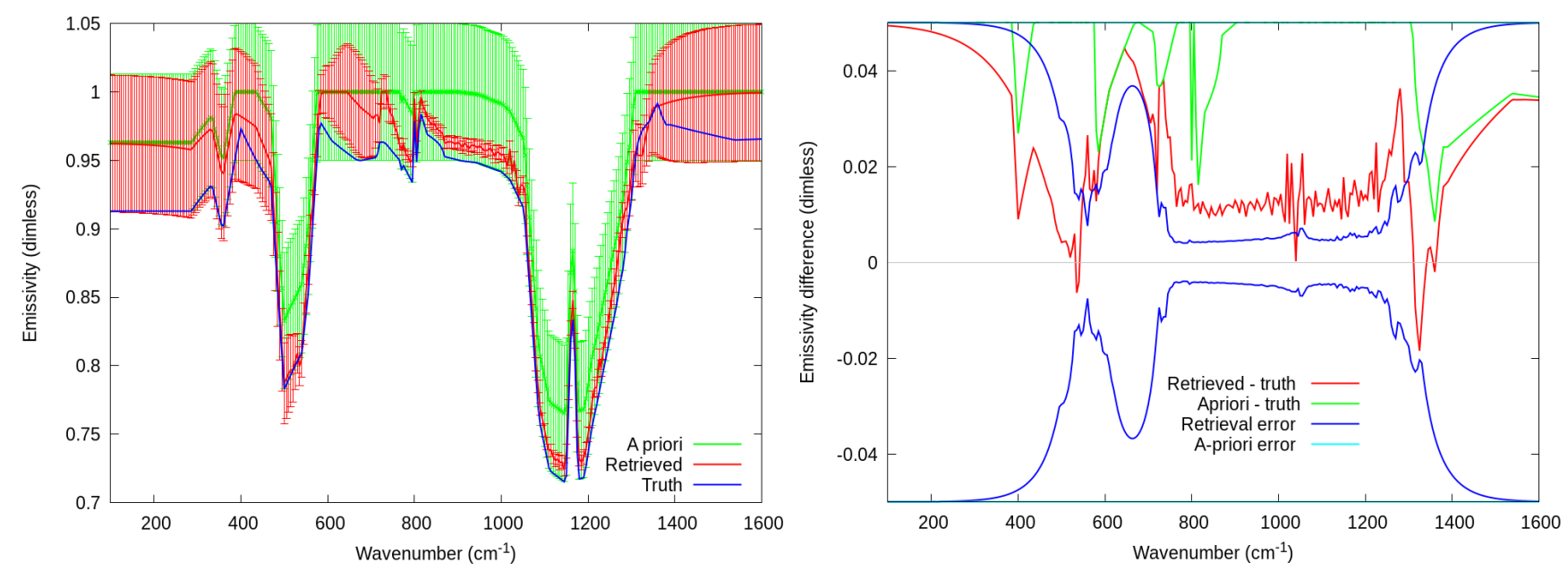

Figure 10. Emissivity retrieval for case 1.1 (desert at noon in summer). Left panel: a-priori profile with error bars (green), retrieved profile with error bars (red), truth (blue). Right panel: difference between retrieved and true (red), retrieval error (blue), difference between a-priori and model (green), a-priori error (cyan).
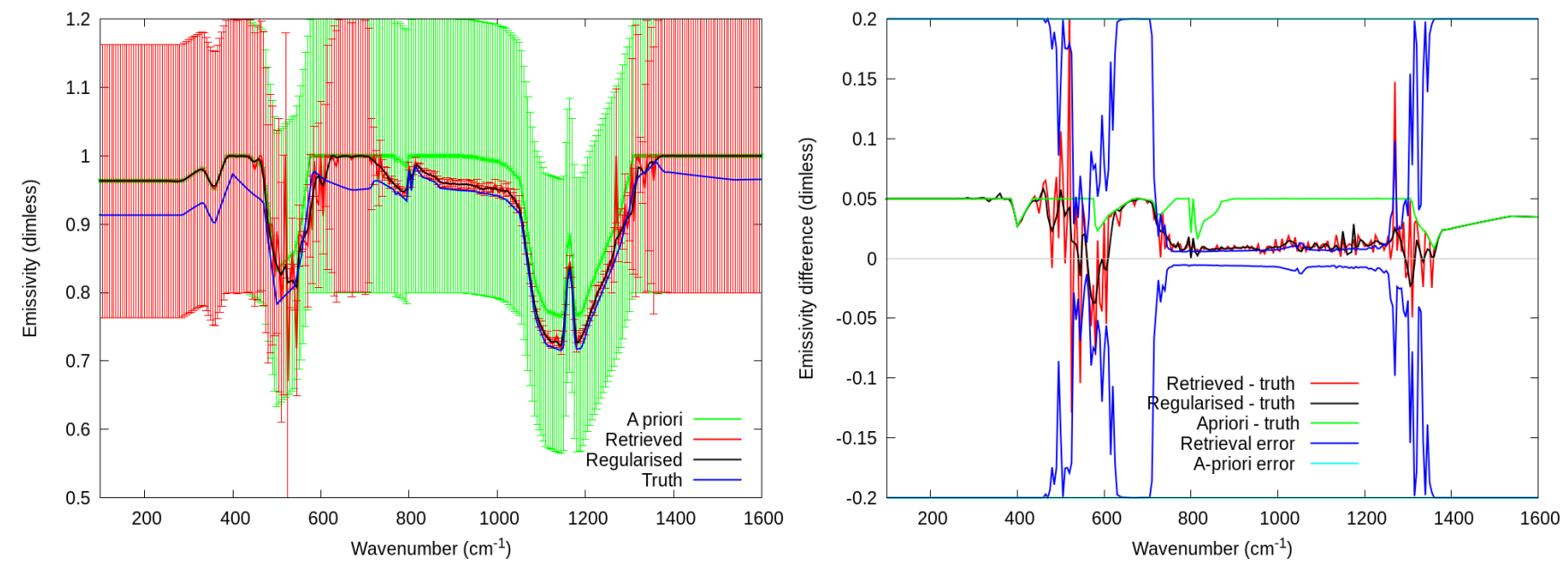

Figure 11. Emissivity retrieval for case 1.1 (desert at noon in summer). A-priori error set to 0.2 and a-posteriori regularization applied. Left panel: a-priori profile with error bars (green), retrieved profile with error bars (red), regularized retrieved profile (black), truth (blue). Right panel: difference between retrieved and true (red), retrieval error (blue), difference between regularized and model (black), difference between a-priori and model (green), a-priori error (cyan). 
settings are under way. In particular the use of a coarser emissivity grid has to be considered. One of the solutions is to use a larger a-priori emissivity error, i.e. 0.2. A larger error of the a-priori however produces oscillations in the retrieved emissivity due to the weaker constrain, which can be reduced by extending the IVS regularization to emissivity. The results are shown in Figure 11.

\subsection{Homogeneous cloudy sky cases}

The second group of tests still deals with the homogeneous case, but for the cloudy-sky.

The quantities retrieved in the cloudy-sky case are: the altitude of the top of the cloud, the equivalent radius of the particle, the total optical depth of the cloud. We realized that there is no sensitivity to the geometrical thickness of the cloud, so we assumed this parameter to be constant. The tests were performed analyzing the simulated E2ES FORUM observations related to the first six homogeneous scenarios described in Table 4 in cloudy sky condition.

\subsubsection{Comparison with SACR code}

The SACR code is able to perform the simultaneous retrieval of the atmospheric state and ice cloud parameters, and it was applied to the analysis of the spectral measurements acquired by the REFIR-PAD spectro-radiometer, which has been measuring at Concordia Station on the Antarctic Plateau since 2012 (Palchetti et al., 2016; Di Natale et al., 2017). The SACR code (Di Natale et al., 2020) performs the retrieval of water vapour and temperature profiles, the surface temperature, the cloud position and and the cloud optical and microphysical properties, such as the generalised ice and water effective diameter, the ice fraction and the optical depth or the IWP. To simulate the atmospheric radiative transfer, the LBLRTM is integrated with a specifically developed subroutine based on the $\delta$-Eddington two-stream approximation, whereas the single-scattering properties of cirrus clouds are derived from a database for hexagonal column habits. To perform the retrieval procedure, SACR code uses the optimal estimation method with the Levenberg-Marquardt approach.

The L2 products obtained from the L2M_I and SACR are here compared. To perform a realistic validation, the atmospheric state used in the retrieval procedure is perturbed with respect to the true state according to the background noise $\mathrm{CM}$, and the atmospheric parameters are not retrieved. Due mainly to the differences in the cloud representation, we cannot truly speak of validation in the cloudy case. Also, the initial guess and a-priori conditions could not be fully replicated in the two codes, because of different code characteristics. The aim of the comparison is to show that the retrieval module of the E2ES and the comparative code have similar capabilities at identifying cloud properties, thus confirming that the results are not code dependent.

\subsubsection{Discussion of the results}

Results for five cloudy cases (the coastal marine case was wrongly attributed by the CIC to a clear sky retrieval due to the small contrast between the cloud and the surface, and case 7 was only studied in clear sky) are summarised in Table 9. 
Table 9. SACR/L2M_I cloudy retrieval comparison.

\begin{tabular}{|c|r|r|r|r|r|}
\hline Cloud Top $[\mathrm{km}]$ & Case 1.2 & Case 2.2 & Case 4.2 & Case 5.2 & Case 6.2 \\
\hline True & 12 & 15 & 10 & 8 & 11 \\
\hline L2M_I & $12.4 \pm 0.01$ & $15.00 \pm 0.05$ & $8.77 \pm 0.02$ & $8.42 \pm 0.01$ & $15.0 \pm 0.05$ \\
\hline SACR & $12.5 \pm 0.26$ & $9.40 \pm 0.03$ & $7.00 \pm 0.06$ & $6.08 \pm 0.02$ & $9.02 \pm 0.06$ \\
\hline \hline Radius $[\mu \mathrm{m}]$ & Case 1.2 & Case 2.2 & Case 4.2 & Case 5.2 & Case 6.2 \\
\hline True & 10 & 6 & 30 & 18 & 80 \\
\hline L2M_I & $10.0 \pm 0.015$ & $5.86 \pm 0.02$ & $28.1 \pm 0.4$ & $17.35 \pm 0.08$ & $55.8 \pm 0.8$ \\
\hline SACR & $10.1 \pm 0.015$ & $4.18 \pm 0.014$ & $26.3 \pm 0.4$ & $18.9 \pm 0.4$ & $86 \pm 5$ \\
\hline \hline Optical Depth & Case 1.2 & Case 2.2 & Case 4.2 & Case 5.2 & Case 6.2 \\
\hline True & 1 & 0.3 & 6 & 3 & 300 \\
\hline L2M_I & $0.94 \pm 0.0006$ & $0.297 \pm 0.0005$ & $5.66 \pm 0.02$ & $3.00 \pm 0.01$ & $9.8 \pm 0.03$ \\
\hline SACR & $0.99 \pm 0.0008$ & $0.496 \pm 0.002$ & $7.66 \pm 0.05$ & $2.88 \pm 0.01$ & $11.98 \pm 0.06$ \\
\hline
\end{tabular}

We see that, qualitatively, the results obtained by the two codes are similar. With some exceptions, the retrieved cloud parameters are very close to the true parameters, even if, being the retrieved parameters characterized by very small errors (given by square root of the diagonal elements of the Covariance Matrix of the retrieval vector), in most cases, the difference between the retrieved and the true value is larger than the error.

In the E2E simulations the cloud composition and scattering properties are the same in the simulation and retrieval of data, so the model error, which is significant with real data and must be accounted for in the Covariance Matrix, does not impact on the retrieval error.

On the other hand, there is a combination of factors that explains the small error bars:

- The error on the assumed atmosphere, which is perturbed with respect to the true state, is not taken into account in the error budget.

- The linear estimate may not be verified when parameters with different units are mixed in the state vector.

- In presence of non negligible correlations in the Covariance Matrix differences between the retrieved value and the true state can be larger than the square root of the diagonal matrix.

There are at least two cases in which the retrieval of cloud parameters is critical also when cloud composition and scattering properties are the same in the simulation and retrieval of data:

- A water cloud at very low altitudes (case 3.2), in which case the cloud is not identified because the cloud and surface effects are similar. 


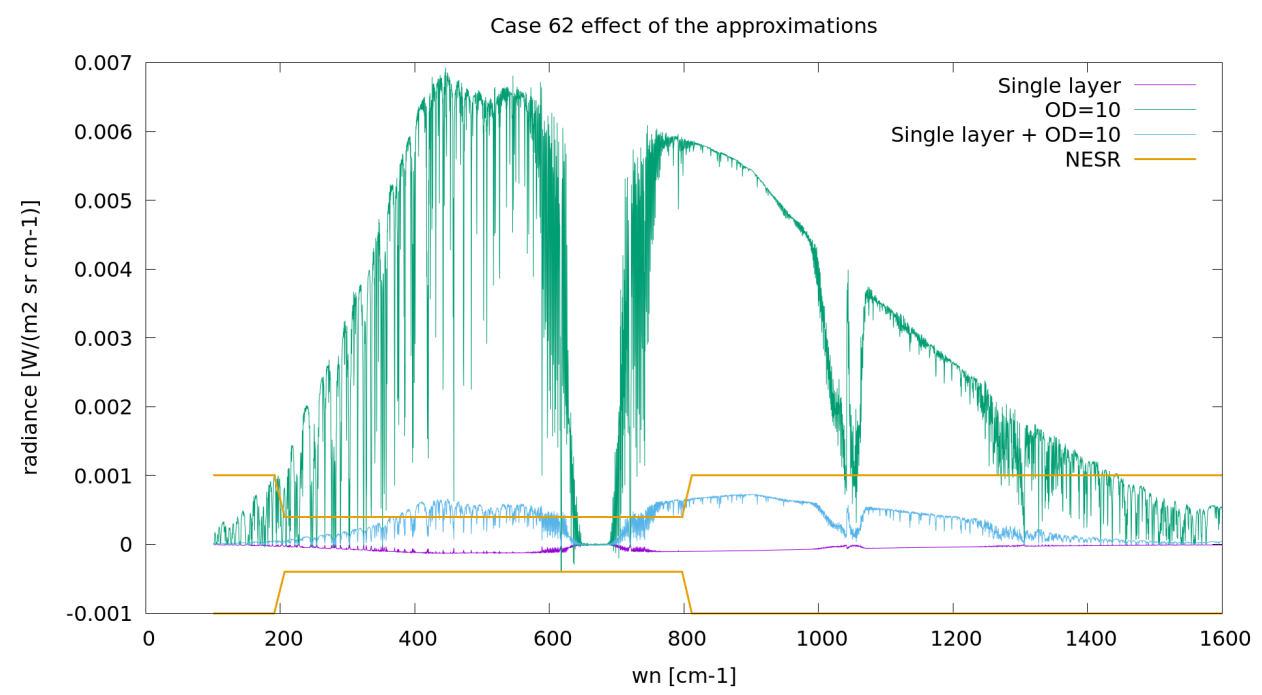

Figure 12. Very thick cloud case (case 6.2, optical depth larger than 100). Quantification of the error in the radiance compared with the noise requirements (yellow curve) using an optical depth of 10 when the cloud is treated either as a single layer (blue curve) or as multiple layers (green curve). The purple curve represents the error when the optical depth of 100 is used and the cloud is represented as a single layer.

- In the case of a cirrus cloud, scattered radiation from below the cloud and direct radiation from above the cloud are mixed in the TOA spectrum. This combination leads to the existence of local minima in the cost function which is minimised by the retrieval procedure. If the initial guess is far from the true values, the retrieval may converge to a local minimum.

For case 6.2, characterized by a very large optical depth, the retrieval values are very far from the true value. However, we verified that for thick clouds, i.e. clouds with optical depth larger than 10 , the cloud becomes fully opaque and the sensitivity to larger optical depths is lost. In this case, the only radiation seen by the instrument comes from above the cloud. Indeed, for thick clouds, two approximations were tested in order to reduce the computing time: maximum optical depth set to 10 and representation of the cloud as a single layer instead of multiple layers. The results are presented in Figure 12.

The optical depth limit of 10, if used alone, introduces an error which is larger than the NESR, because some radiation however penetrates the first layers of the cloud. The error becomes smaller than the NESR if also the single layer approximation is applied, because the compactness of the cloud avoids the penetration of the radiation. Indeed, most of the reduction in the computation time comes from the single layer approximation alone, and the effect of this optimization on the spectrum is negligible, if no limit on the optical depth is used. Thus, the tests were performed with these settings. Of course, there is still no sensitivity to large values of the optical depth. 
Table 10. Cirrus cloud cases 1.2 (cirrus on desert) and 2.2 (cirrus on ocean). Minimum optical depth that can be detected when either all FORUM spectrum is used (FIR+MIR) or only the MIR part $\left(667-1100 \mathrm{~cm}^{-1}\right)$ is used.

\begin{tabular}{|c|r|r|}
\hline Case \# & FIR+MIR & MIR \\
\hline 1.2 & 0.1 & 0.1 \\
\hline 2.2 & 0.03 & 0.05 \\
\hline
\end{tabular}

\subsubsection{Sensitivity to thin cirrus cloud}

545 The far-infrared part of the spectrum is particularly sensitive to cirrus clouds and recently, simulations were used to show that FIR can contribute to improve the detection of thin cirrus cloud (Maestri et al., 2019b, a; Magurno et al., 2020). The analysis with the E2E simulator allows to evaluate the capability of retrieving cloud information from these measurements also in presence of very thin cirrus clouds and to compare the lowest detectable optical depth for a cirrus cloud by FORUM (range $100-1300 \mathrm{~cm}^{-1}$ ) with the one obtainable from the analysis of Middle InfraRed (MIR) measurements only (range 667-1300

$\mathrm{cm}^{-1}$ ). The sensitivity of the retrieval to the optical depth of the cirrus cloud depends on the contrast between the surface and the cloud and this depends on the characteristics of the surface and the atmosphere. Two cases were considered for this analysis: cirrus on the desert, and cirrus on the sea, see Table 5. The surface properties are assumed homogeneous for the entire scene.

For both cases, we started from the optical depth value of the considered case and then we progressively halved the optical depth whilst keeping all other parameters unchanged until the Cloud Identification and Classification tool continued to classify this case as cloudy. Then L2M_I retrieval was performed to be sure that the retrieval was able to retrieve the cloud parameters. Contrary to previous tests where perturbed values of the truth were used for water vapour, temperature and surface temperature, for these tests they were set to the true values to avoid that a different error in temperature and water vapour had a different impact in the retrievals performed in the two different spectral regions. The results for the two considered cases are reported in Table 10.

For case 1.2 the minimal optical depth was 0.1 for both MIR and FIR+MIR, while for case 2.2 the minimal optical depth was 0.05 when only MIR measurements are used and 0.03 when both FIR and MIR bands are combined. The addition of the FIR band reduces the retrieval error and enhances the accuracy of the retrieval.

Figure 13 reports the differences between the cloudy spectra relative to various optical depths and the clear-sky spectrum, compared with the random measurement error. Clouds are detectable and cloud parameters retrievable until the contribution of the cloud to the spectrum is larger than the noise. In real life, where the knowledge of the atmospheric profiles is affected by an error and in presence of other systematic errors, the retrieval of the cloudy quantities may be more difficult, but the information provided by this test represents the goal we can aim to reach. 

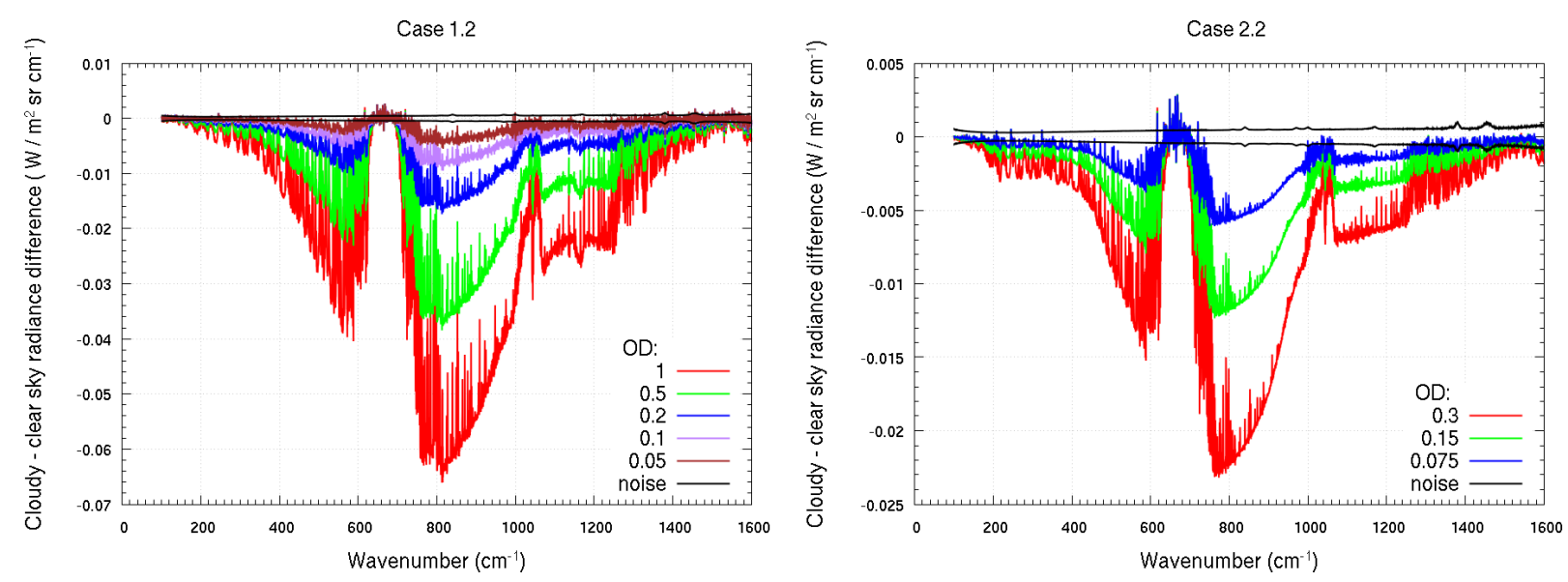

Figure 13. Difference between the TOA radiance in cloudy case for various optical depths of the clouds and the clear sky case of case 1.2 (left panel) and case 2.2 (right panel), compared with the measurement error

\subsection{Tests on heterogeneouos cases}

Given the extension of the FSI pixel, the probability that the scene might be heterogeneous is very high. Heterogeneity of the pixel poses different problems in the retrieval of L2 products (either atmospheric profiles, surface temperature and emissivity or cloud information) from TOA radiance. The first issue is that the ISRF is modelled assuming a homogeneous FoV. If the FSI FoV is strongly heterogeneous, the ISRF may not well represent the instrumental response, thus introducing an error in the retrieval. In the case in which some predominant homogeneous characteristics can be identified in the heterogeneous scene, it is useful to quantify the error in the retrieval of the predominant homogeneous contribution due to the contamination of the scene with clouds or heterogeneities in the surface. On the contrary, if the scene is so heterogeneous that predominant homogeneous characteristics cannot be identified, the measured spectrum has a value in itself, but the information extracted by the L2 analysis is just a combination of contributions coming from the different parts of the sounded atmosphere and surface. The different types of heterogeneities that can occur in the pixel is manifold and the impact of heterogeneities in different scenarios can be very different. A large effort was made in the development of the SGM to handle heterogeneous scenes, primarily to study the impact of these heterogeneities on the spectrum and then on the retrieved quantities. In the standard approach used by the L2M module the pixel is assumed to be homogeneous, and the retrieval is performed in either clear sky or cloudy sky condition on the basis of the CIC estimates. Thus, at the moment, we regard any heterogeneity of the FSI FoV as a contamination of either a clear or a cloudy homogeneous scene.

\subsubsection{Heterogeneities in the FSI FoV: convolution with ISRF}

The objective of this test is to check whether the ISRF, which is modelled assuming a homogeneous FoV, allows a good representation of the radiance resulting from the OSS also in presence of heterogeneities in the FSI FoV. The FSI is able to 

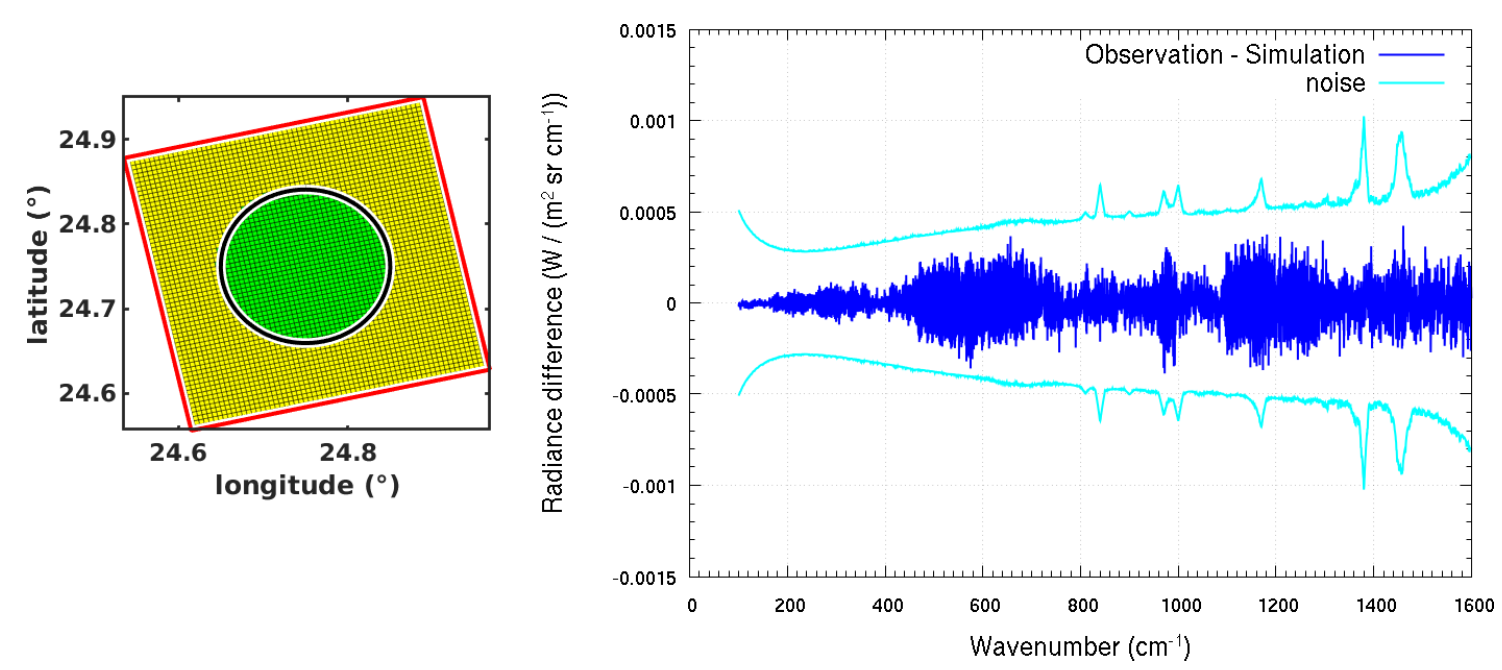

Figure 14. Left panel: FORUM FoV: FEI FoV (yellow), FSI FoV: clear (green). Right panel: difference between the output radiance of the FSI and the simulation of the OSS radiance by L2M by convolution of the high resolution with the ILS, compared with measurement noise, in the homogeneous case.

accurately reconstruct the radiance seen by the instrument taking care of the different beams coming from different parts of the FoV. L2M simulates the spectrum observed by the instrument by convolving the high resolution spectrum generated by the forward model with the ISRF computed assuming a homogeneous FoV, and hence the deformations in the ISRF introduced by the heterogeneities are neglected. This may have a large impact on the quality of the retrieval, since the information on the profiles at the different altitudes comes from the knowledge of the line shapes of the spectral lines. The magnitude of the impact depends on the heterogeneities which are considered. Given the cylindrical symmetry of the FSI, heterogeneities in the FoV that are expected to mostly affect the ISRF are the ones that have a strong dependence on the radius.

To this purpose, we performed the following test.

1. In the homogeneous case 1.1 we considered the spectrum calculated by the OSS and the one obtained by convolving the high resolution spectrum generated by SGM with the ISRF. We compared the difference between these two spectra with the error noise.

2. We repeated the same comparison, this time using a cirrus covering only a part of the pixel in the center of the FoV. In this case we used the SGM high resolution averaged spectrum, convolved with the ISRF and compared with the OSS spectrum.

Figures 14 and 15, show, respectively for the homogeneous case and the heterogeneous one, the FSI FoV and the difference between the radiance outgoing the FSI with the radiance computed by convolving the high resolution spectrum computed by the forward model with the ISRF. This difference is plotted as a function of the wave number, together with the measurement noise. 

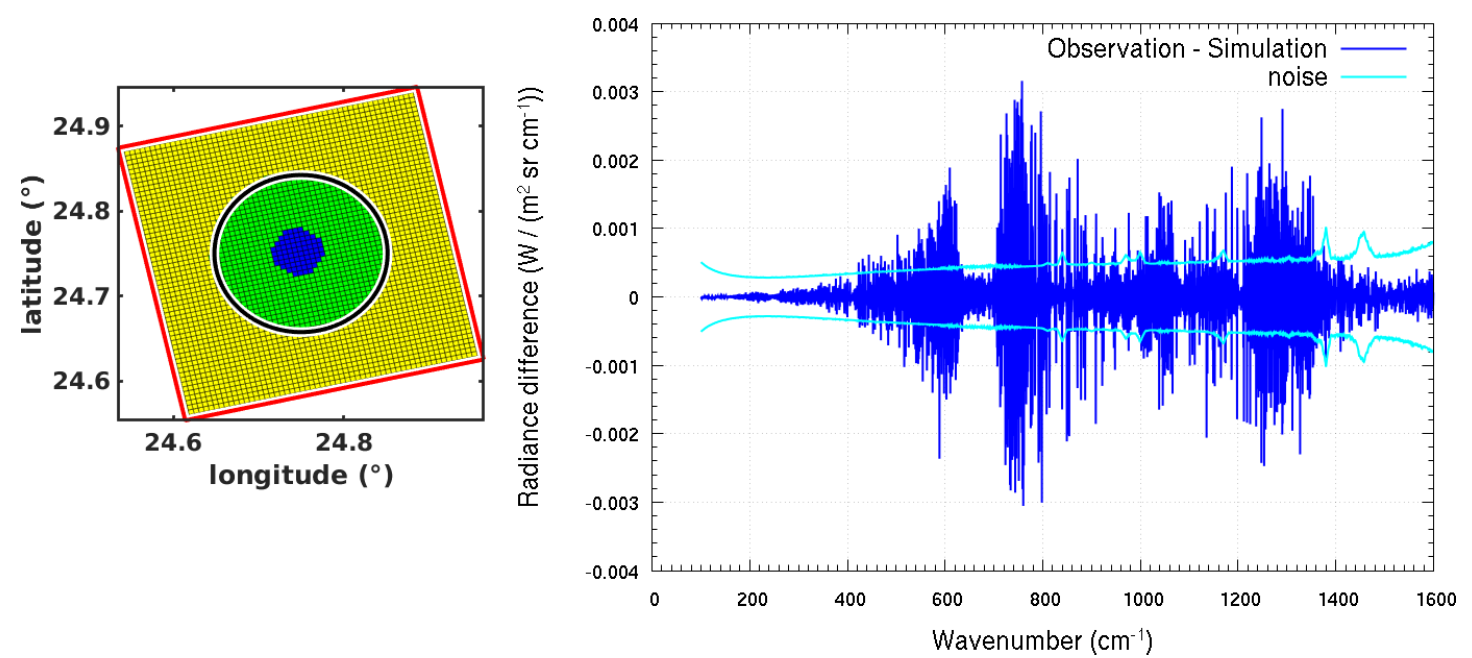

Figure 15. Left panel: FORUM FoV: FEI FoV (yellow), FSI FoV: clear (green) and cloudy (blue). Right panel: difference between the output radiance of the FSI and the simulation of the OSS radiance by L2M by convolution of the high resolution with the ILS, compared with measurement noise, in the heterogeneous case.

In case of a homogeneous scene the convolution with the ISRF is a good representation of the instrumental response. On the contrary, when strong heterogeneities are present, the convolution with the ISRF introduces an error larger than the measurement noise. In principle, the error coming from the representation of the ISRF cannot be neglected in presence of large discontinuities as the ones we have considered. However, the heterogeneity of the scene poses anyway problems to the retrieval module, which considers the scene to be homogeneous. Also, the meaning of the retrieved quantities is uncertain since the measured spectrum represents a kind of average of the different scene characteristics.

In the future, the L2M approach can be developed to use information coming from FEI image and chi-square to identify and define dedicated strategies for heterogeneouos scenes.

\subsubsection{Cloud contamination of clear sky FoV}

615 This test aims at estimating the impact that an unidentified cloud contamination of the FSI FoV has on the retrieval, performed with the assumption of clear sky conditions, of temperature and water vapor profiles, and of the surface temperature and frequency dependent emissivity. The test is performed by simulating with the SGM a clear sky scene, which is progressively contaminated by a cloud entering in the FSI FoV. This is the most simple and idealized case, with only two distinct FoV fractions internally homogeneous, that is investigated as a reference case. In real conditions, larger heterogeneities can be observed within the FoV. For this specific test, the study case 1.1/1.2 (tropical profile on desert surface) is used. The parameters of the cloud which contaminates the clear-sky scene correspond to an optically thin cirrus cloud: top $=12 \mathrm{~km}$; thickness $=2 \mathrm{~km}$; $\mathrm{OD}=1 ;$ Reff $=10 \mu \mathrm{m}$. The surface properties, as well as the atmosphere, are assumed homogeneous for the entire scene. The 

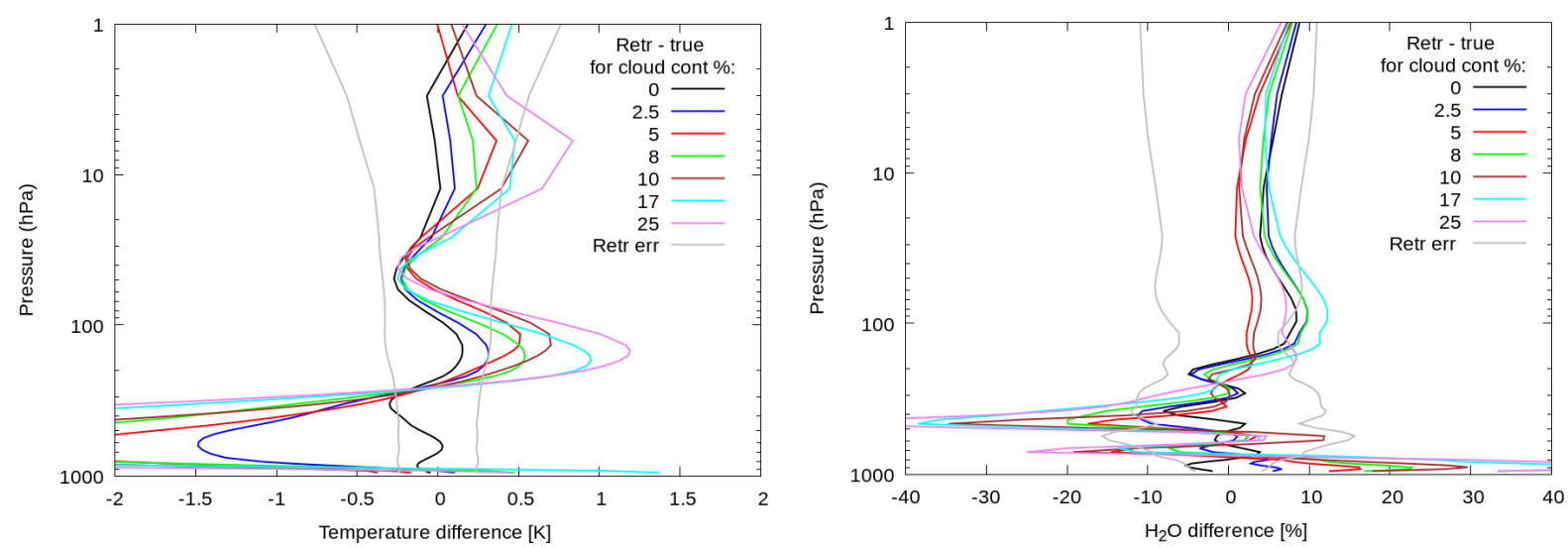

Figure 16. Differences between retrieved and true profiles for temperature (left panel) and water vapour (right panel) for different percentage of cloud contamination in the FSI FoV, compared with the retrieval noise.
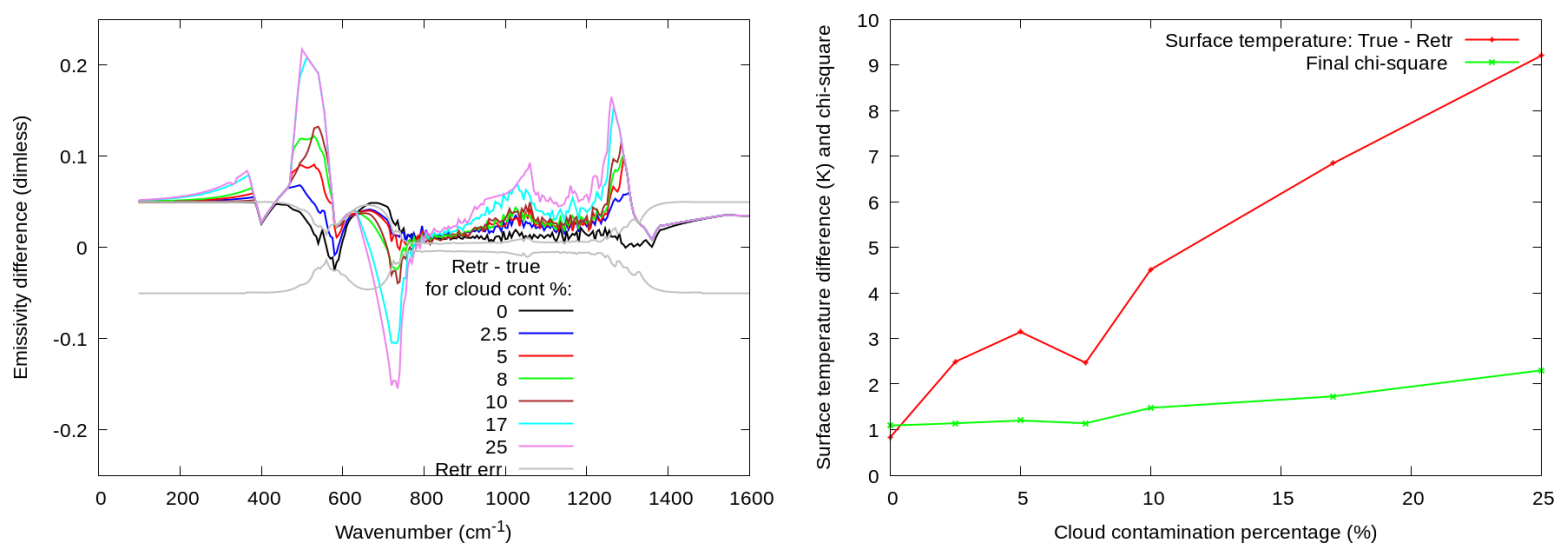

Figure 17. Right plot: Difference between retrieved and true values of surface emissivity for various percentage of cloud contamination in the FSI FoV, compared with the retrieval noise. Left plot: Differences between true and retrieved value of surface temperature for various percentage of cloud contamination in the FSI FoV, compared with the retrieval noise. The final chi-square values are also reported.

FoV fraction occupied by the cloudy sector is increased at steps of $2.5 \%$ from 0 to $10 \%$, then at steps of about $7 \%$ up to $25 \%$ cloud coverage fractions.

The retrieval is performed for each simulation by assuming clear sky conditions, and the retrieved quantities are compared with the values used by the SGM to simulate the part of the scene in clear-sky. These are indicated as the true values. Figure 16 shows the differences between true and retrieved values for the temperature profiles (left panel) and the water vapor profiles (right panel), compared with the corresponding retrieval error for different cloud contamination percentages. In general, increasing the cloud contamination increases the difference of the retrieved temperature profile with respect to the true expected 
Table 11. Retrieved surface temperature for different percentages of water/snow FoV coverage.

\begin{tabular}{|c|l|l|r|}
\hline Case & $\begin{array}{l}\text { Percentage of pixel surface } \\
\text { covered with snow }\end{array}$ & $\begin{array}{l}\text { Percentage of pixel surface } \\
\text { covered with water }\end{array}$ & Retrieved surface temperature \\
\hline 1 & 10 & 90 & $270.3 \pm 0.4$ \\
\hline 3 & 25 & 75 & $263.0 \pm 0.4$ \\
\hline 2 & 55 & 45 & $265.9 \pm 0.4$ \\
\hline 4 & 90 & 10 & $260.9 \pm 0.4$ \\
\hline
\end{tabular}

values. In the worst case, at about $700 \mathrm{hPa}$, the differences increase from within the error for the completely clear sky case (red line) up to about six times larger than the error for the case $97.5 \%$ clear $-2.5 \%$ cloudy (black line). Similar results, but with a reduced magnitude, are observed for the water vapor profile retrieval. The surface skin temperature retrieval is also affected by the cloud contamination. Figure 17 shows the difference between the true and the retrieved values of the surface emissivity as a function of the clear sky FoV fraction (red line) and the corresponding $\chi^{2}$ values (green line). The differences in the retrieved temperature rapidly increase up to $3 \mathrm{~K}$ for a $5 \%$ cloud contamination in the tested scenario. As described in Subsection 5.1.2 a negative bias in the surface temperature is compensated by a positive bias in the surface emissivity. Since the considered cloud is an optically thin cirrus cloud ( $\mathrm{OD}=1$ ), we expect that for thicker clouds, such as in case of cumuli or cumulonimbi, the effects on the observed radiance and hence on the retrieved skin temperature are larger even for lower fractional areas. For the tested scenario, the quality of the retrieval is compliant with the E2ES requirements for a cloud contamination smaller than $10 \%$. Nevertheless, the CIC algorithm applied to the simulations identified the scene as cloudy as soon as the cloud fraction grew above $8 \%$. This means that the automatic inversion procedure would switch to the cloudy case and would not try to retrieve the surface properties in the worst scenarios, where the errors are too large, preserving the performance of the L2M_I.

\subsubsection{Clear sky, heterogeneities in the surface}

This test aims to evaluate the impact of the heterogeneity of the surface on the retrieval of temperature and water vapour profiles. It is performed for a polar scene, since the largest information on the surface is obtained mainly in the polar regions where the atmosphere is drier and hence more transparent. The scene at the geolocation corresponding to case 5.1 (polar case) is simulated assuming that the surface has a part covered with snow and a part covered with sea. The surface temperature used to generate observations changes accordingly, being $273.27 \mathrm{~K}$ on water and $258.055 \mathrm{~K}$ on snow. Temperature and water vapour profiles are assumed homogeneous in all the sounded atmosphere over the pixel. Results are shown for scenes covered with different percentages of water and snow.

Figure 18 reports the retrieved emissivity in the various cases, while the retrieved surface temperature is reported in Table 11. 


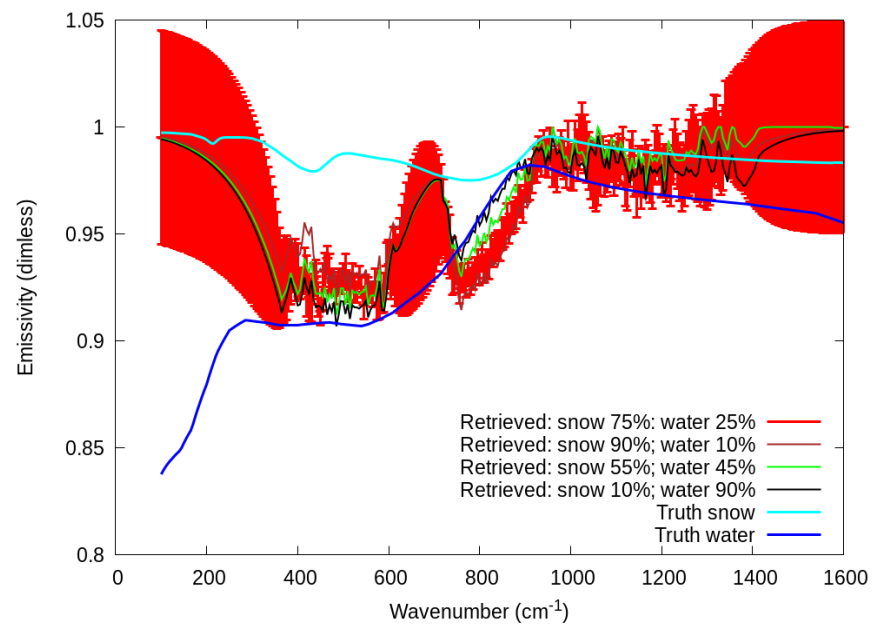

Figure 18. Retrieved profiles for different types of soil heterogeneity for emissivity, and true profiles for homogeneous snow (cyan) and water (blue) solis

In all cases we find that retrieval of surface emissivity, as well as surface temperature, change for the different cases, with retrieved values in the spectral region between 400 and $600 \mathrm{~cm}^{-1}$, where water and snow emissivity mostly differ, being always closer to the water surface emissivity despite the percentage of pixel coverage of the water surface. No bias is introduced by the heterogeneous pixel in the retrieval of water and temperature profiles, since the differences between the retrieved value and the true one are always within the retrieval error (see Figure 19).

\subsubsection{Realistic tests on MODIS data}

All the previously investigated scenarios rely on idealized conditions, where the surface and, eventually, the cloud layer can only have homogeneous properties within the FSI FoV or a simple binomial characterization. This simplified scene description is convenient to evaluate study case scenarios and to guarantee a good computational speed of the E2ES chain. Unfortunately, the real world is far from being ideal and almost homogeneous conditions are more likely the exception rather than the rule. Surface, atmosphere, and cloud properties can significantly vary on spatial scales of the order of few kilometers or less, and their effects combine into a single $15 \mathrm{~km}$ diameter FoV FSI observation.

This last test aims at evaluating the E2ES performance in case a realistic, largely heterogeneous scene is ingested by the L2M module. Three complex case studies are generated with the SGM: a clear sky case (M.1) over land, where the scene heterogeneity depends on the surface properties (temperature and emissivity) and on the atmospheric profiles (temperature and water vapor concentration); two cloudy sky scenes over land (M.2) and sea (M.3), where the clouds inhomogeneities sum up with those of surface and atmosphere. Close locations in southern England are selected, whose geolocation and time are reported in Table 12. 

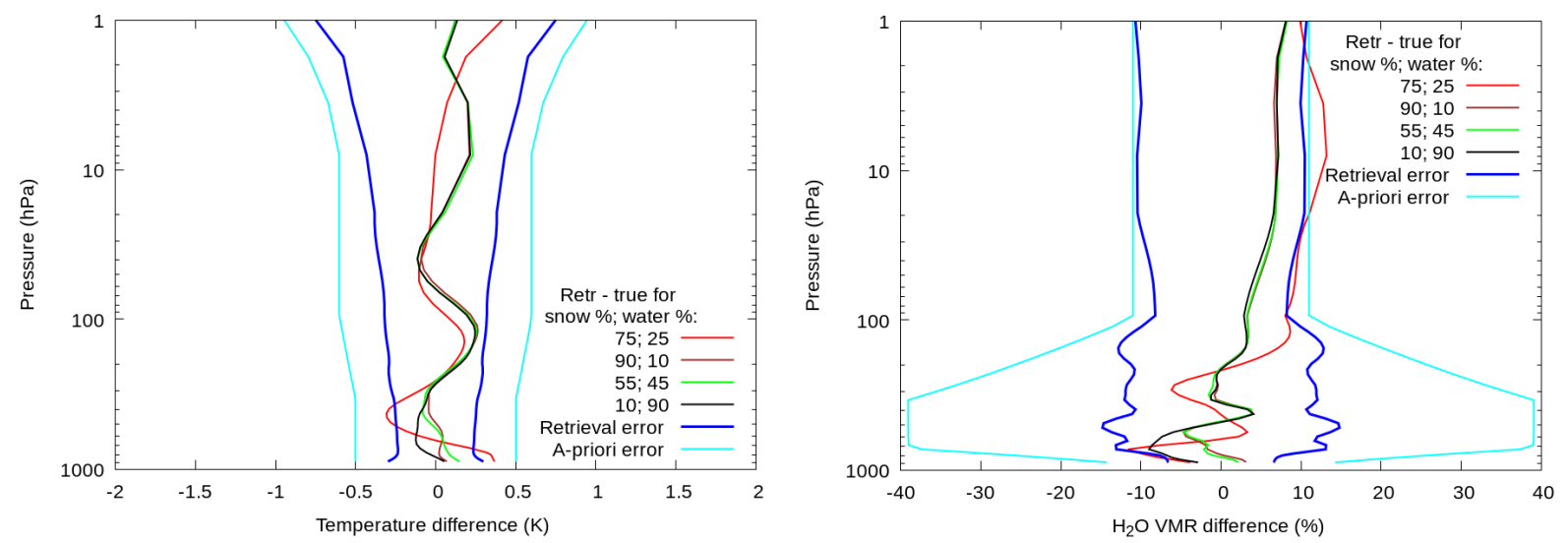

Figure 19. Differences between retrieved and true profiles for different types of soil heterogeneity for temperature (left plot) and water vapour (right plot). Retrieval and a-priori errors are also shown.

Table 12. Scene definition for heterogeneous MODIS cases.

\begin{tabular}{|c|l|l|l|}
\hline Case \# & Geolocation & Time & Description \\
\hline M.1 & $52.70^{\circ} \mathrm{N} / 1.85^{\circ} \mathrm{W}$ & 7 Feb $2018,13: 30$ & Clear sky over land \\
\hline M.2 & $53.50^{\circ} \mathrm{N} / 2.00^{\circ} \mathrm{W}$ & 7 Feb $2018,13: 30$ & High cirrus clouds over land \\
\hline M.3 & $51.38^{\circ} \mathrm{N} / 4.15^{\circ} \mathrm{W}$ & 7 Feb $2018,13: 30$ & High cirrus clouds over sea \\
\hline
\end{tabular}

The necessary parameters to build the scene are derived from the following databases, homogenized and remapped into the FEI grid:

- Surface: the Global Land Cover Map (GlobCover 2009, http://due.esrin.esa.int/page_globcover.php) is an ESA's composite product based on MERIS (Medium Resolution Imaging Spectrometer Instrument) data collected at $300 \mathrm{~m}$ spatial resolution. The GlobCover map provides 22 land cover classes, defined with the United Nations land cover classification system, that are associated to the 11 surface types from Huang et al. (2016) used in the SGM surface definition;

- Atmosphere: the MODIS Atmospheric Profile product (07_L2, https://modis-images.gsfc.nasa.gov/products.html) defines the temperature and water vapor profiles, and the surface height at $5 \mathrm{~km}$ resolution;

- Clouds: the MODIS Cloud Product (06_L2) are used to define the cloud parameters (particles phase and effective radius, cloud top height and optical thickness) and the surface temperature, with spatial resolution of $1 \mathrm{~km}$. 

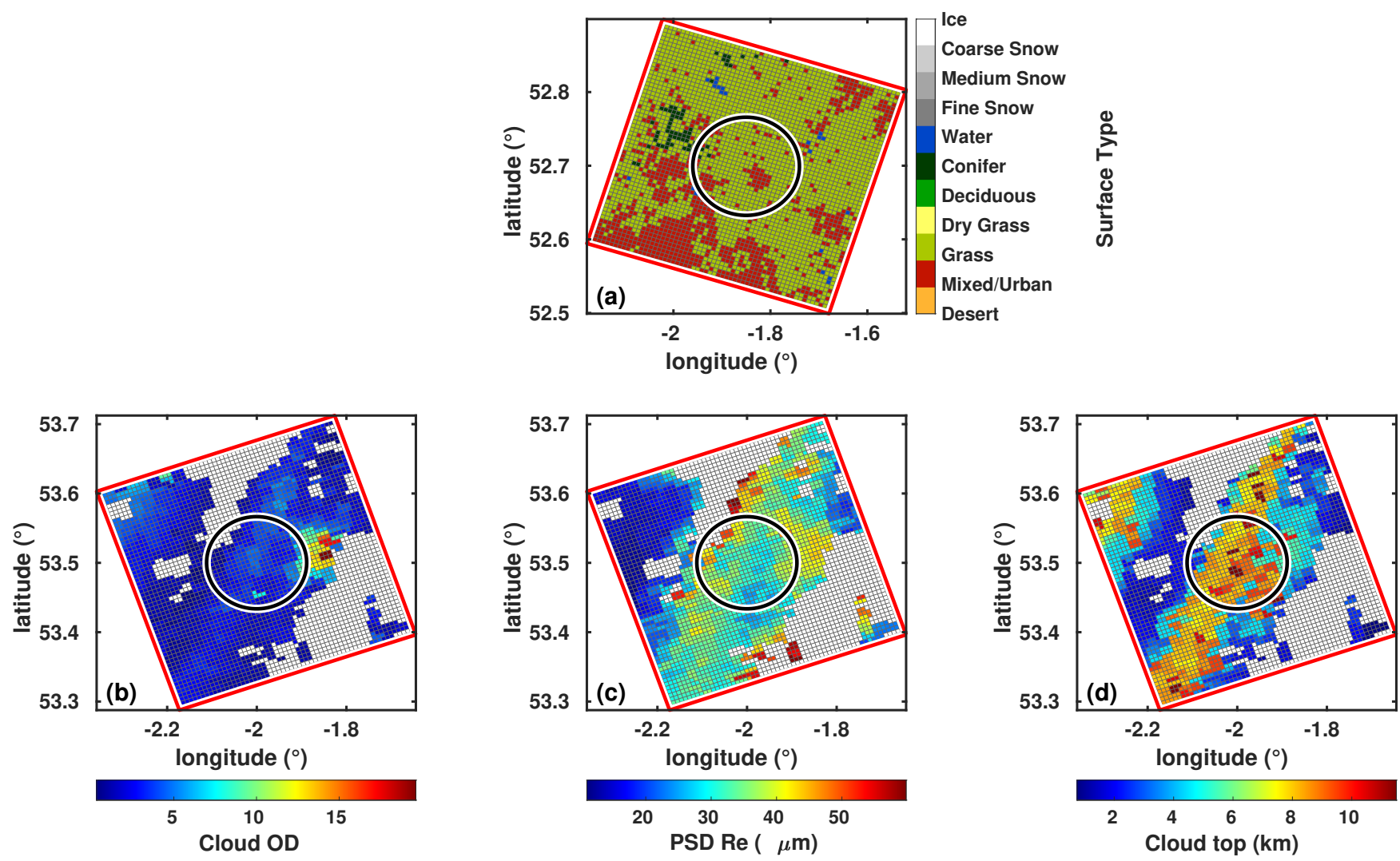

Figure 20. Top panel: Surface type for the study case M.1, derived from GlobCover database and remapped on the FEI grid. The FSI FoV is marked with the black ring. Bottom panels: Cloud optical depth (left), PSD effective radius (center), and cloud top height (right) for the study case M.2, derived from MODIS L2 data and remapped on the FEI grid. White areas indicate clear sky.

Note that the SGM is not designed to build such complex scenes for the standard E2ES configuration. These scenarios are built with a dedicated subroutine and stored in the E2ES chain for the testing activities only.

Figure 20 shows few examples of the surface and cloud parameters remapped into the FEI grids. The scene observed in the M.1 clear sky case (top panel) has irregularly sparse urban areas (brown) surrounded by different kinds of vegetation (light and dark green) and few wet areas (blue, probably lakes or ponds), creating a much more complex scenario than an ideal homogeneous case. Similarly, clear sky areas alternate to high and low level clouds, with unevenly distributed optical and microphysical parameters, in the cloudy case M.2 (bottom panels). Thus, multiple spectral radiances are computed by the SGM for all the FEI grid points within the FSI FoV (the black ring in the Figures), according to the different properties of the scene. Then, the radiances are linearly combined to produce a single spectral radiance to feed the FSI-OSS module. Finally, the L2M is used to retrieve the characterizing parameters of the complex scenes, in particular: temperature and water vapour 
Table 13. Cloud parameter results for cloudy MODIS cases M.2 and M.3.

\begin{tabular}{|c|l|l|}
\hline Property & Reference & Retrieved \\
\hline \multicolumn{3}{|c|}{ Case M.2 } \\
\hline Cloud Top Height & $2 .-11.5$ & $9.00 \pm 0.07$ \\
\hline Optical Depth & $0.51-9.66$ & $2.04 \pm 0.04$ \\
\hline Particle Size & $24.3-50.7$ & $67.11 \pm 0.08$ \\
\hline \multicolumn{3}{|c|}{ Case M.3 } \\
\hline Cloud Top Height & $7.0-11.5$ & $10.04 \pm 0.03$ \\
\hline Optical Depth & $0.74-3.98$ & $1.44 \pm 0.06$ \\
\hline Particle Size & $10.9-23.7$ & $31.95 \pm 0.05$ \\
\hline
\end{tabular}

profiles, and surface skin temperature and emissivity for the case M.1, correctly classified by CIC as a clear sky scene; cloud properties for the cases M.2 and M.3, classified by CIC as cloudy scenes.

In the analysed realistic cases, it is not possible to define a single truth to be compared with the retrieval results. The average parameters within the FSI FoV do not properly describe the scene as a whole. To evaluate the L2M_I performance, the assessment test verifies that the retrieved scalar quantities lie within one standard deviation from the average value, and that spectral quantities and profiles are consistent, within the error bars, with the bulk of the input values.

Figure 21 shows the retrieval results (black curves) of the clear sky case M1. Temperature and water vapor profiles (upper panels) are compared with all the reference profiles that are considered for the SGM simulation within the FSI FoV, derived from the MODIS L2 data. The retrieved profiles are consistent with the input profiles along the entire vertical extension of the model. The bottom panel shows the emissivity retrieval. As already shown in Section 5.1.2, the sensitivity to surface emissivity at mid-latitudes is limited to the spectral range $700-1300 \mathrm{~cm}^{-1}$, because of the large absorption of water vapor in the FIR. We show the emissivity profiles used by the SGM along with the percentage of FoV pixels. The retrieved emissivity in the atmospheric window is very close to the weighted average of the input profiles, suggesting the capabilities of the inversion procedure in a complex scenario. Finally, the model surface skin temperature of $275.96 \pm 0.05 \mathrm{~K}$ is well reconstructed by the retrieved value $276.12 \pm 0.39$.

Table 13 shows the retrieval results for the two cloudy sky cases, M.2 and M.3 respectively. The retrieved cloud parameters (optical depth, PSD effective radius, and cloud top height) are scalar values, therefore, they are compared with the range of the same parameters for all the FSI FoV subpixels. As in the previous case, despite the heterogeneity of the observed scene, the $\mathrm{L} 2 \mathrm{M}$ retrieves values that are in the range of the observed scene. There is a slight tendency to overestimate the particle radius, but for large particles there is no sensitivity in the measurements. These tests further confirm the good performance of the E2ES as a tool for the analysis of ideal and realistic study cases. 
https://doi.org/10.5194/amt-2021-196

Preprint. Discussion started: 2 September 2021

(c) Author(s) 2021. CC BY 4.0 License.
Atmospheric

Measurement

Techniques

Discussions
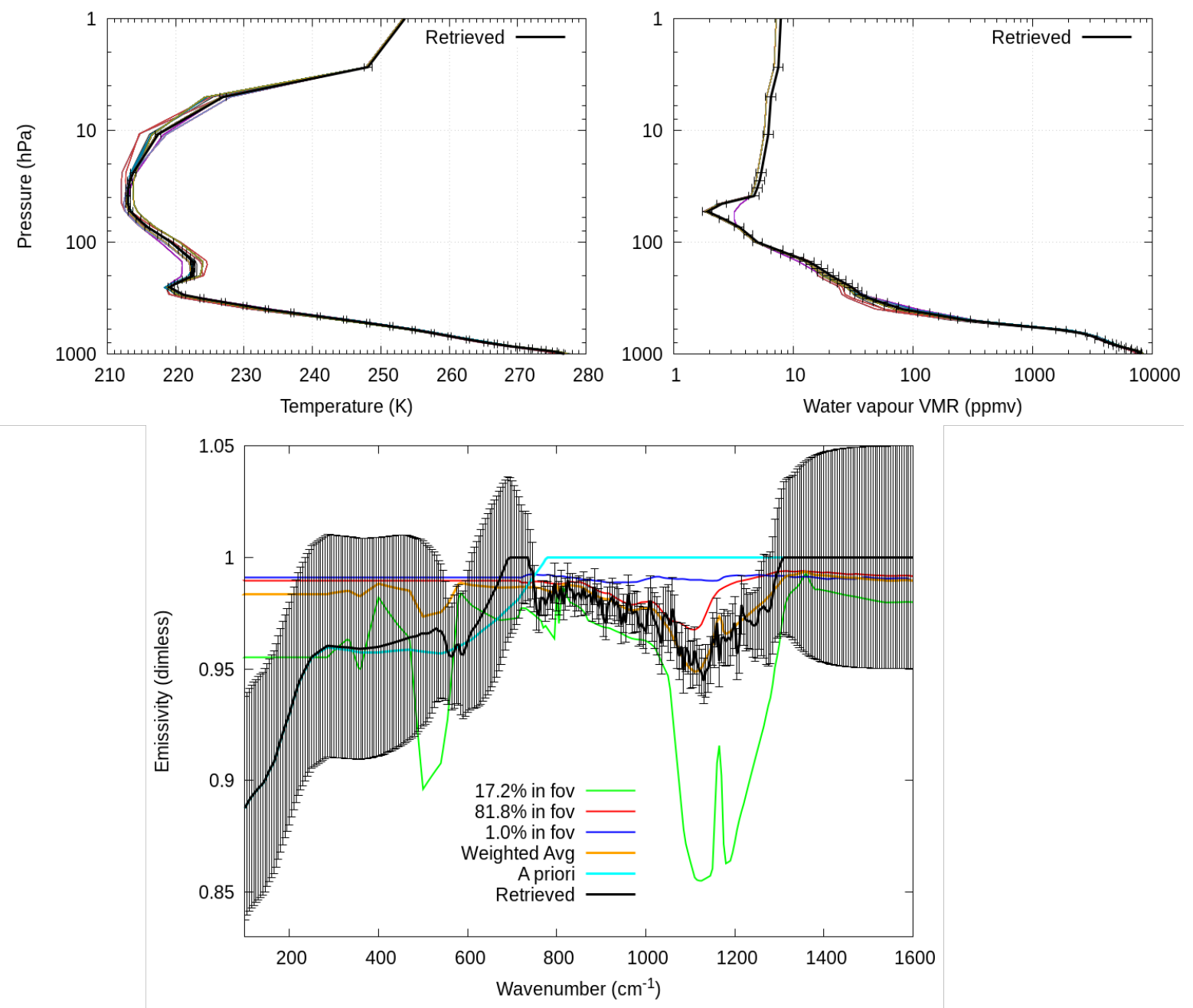

Figure 21. Comparison between the retrieved parameters and the input reference ones for the clear sky case M.1. Top left, temperature profiles for the FoV pixels and retrieved profile with retrieval error. Top right, water vapor profiles for the FoV pixels and retrieved profile with retrieval error. Bottom, emissivity profiles with percentage in the FoV pixels, a priori, weighted emissivity average and retrieved profile with retrieval error. 


\section{Conclusions}

The findings of the FORUM E2ES project are presented. In the E2E the geometry of the orbit, the atmosphere and the FSI and FEI instruments are fully modeled with realistic configurations and retrieval is performed assuming either full clear sky or cloudy-sky scenes.

We first successfully validate the performances of the E2E chain with correlative codes, a necessary step to ensure that there are no flaws. Then, we select some homogeneous test cases, both in clear and in cloudy sky, chosen to represent all different atmospheric conditions, soil characteristics and cloud types. In all cases, the retrieved quantities satisfy FORUM requirements. In cloudy sky, we also show that the optical depth threshold for a cirrus to be detected varies between 0.03 and 0.1 , with generally better sensitivity if the FIR is included. The threshold depends on the different characteristics of the surface and the atmosphere.

The influence on the retrieval of inhomogeneities in the field of view is also investigated. The convolution of the high resolution spectrum with the ISRF function (computed assuming a model homogeneous FoV) well represents the instrumental effects for a homogeneous FoV, with the error being below the NESR threshold. This is not the case when a strong inhomogeneity is considered.

Heterogeneous soil in the FoV does not impact the retrieval of atmospheric profiles, with the retrieved surface temperature and emissivity approaching the weighted averages of the surface properties.

Even a small contamination of a cloud in the FoV induces errors in the retrieved atmospheric and surface quantities. The error increases with the increase of the percentage of the FoV affected by the contamination and of the optical depth of the cloud.

Finally we perform the retrieval on some realistic, heterogeneous scenes, which, however, present a predominance of either clear sky or cloudy sky. We show that the retrieval converges to values that are included in the range of variability of the quantities used to model the radiances.

The E2E chain has been used to show how the instrument characteristics and scene conditions impact on the spectrum measured by the instrument, and the consequences on the retrieval process. Critical issues have been highlighted.

735 These issues should be addressed both by developing a more sophisticated retrieval approach, and by exploring the best way to use the IASI-NG sinergy.

Author contributions. All authors contributed to the writing of this paper.

- Claudio Belotti wrote the L2M python wrapper.

- Maya Ben-Yami performed some tests.

- Giovanni Bianchini was responsible for the OSS module, and wrote the FSI code.

- Bernardo Carnicero Dominguez was the FORUM Phase A study manager.

- Ugo Cortesi coordinated the contacts between CNR and DEIMOS. 
https://doi.org/10.5194/amt-2021-196

Preprint. Discussion started: 2 September 2021

(C) Author(s) 2021. CC BY 4.0 License.

(c) (i)

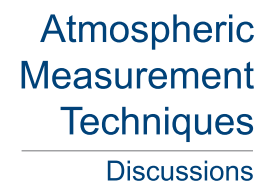

- Samuele Del Bianco was responsible for the E2E validation, and performed the tests in clear sky.

- Gianluca Di Natale performed the validation tests in cloudy sky.

- Tomás Guardabrazo was the E2E project manager, contributed the geometry module and wrote the FEI and PAM modules.

- Dulce Lajas was the E2E FORUM ESA's Technical Responsible during the FORUM Phase A and Phase B1.

- Tiziano Maestri was responsible for the SGM module.

- Tiziano Maestri, William Cossich and Davide Magurno wrote the SGM and L2M_CIC code.

- Hilke Oetjen is the FORUM Mission Scientist.

- Piera Raspollini was responsible for the assessment campaign, and performed most of the tests of this paper.

- Cristina Sgattoni performed some tests.

- Luca Sgheri was responsible for the L2M module, wrote the L2M inversion code and coordinated the writing of this paper.

Competing interests. No competing interests are present

Acknowledgements. We would like to thank all the FORUM research group, a large and active community. Only by working together the FORUM project grew from an instrument concept to an operative mission. 
https://doi.org/10.5194/amt-2021-196

Preprint. Discussion started: 2 September 2021

(C) Author(s) 2021. CC BY 4.0 License.
Atmospheric

Measurement

Techniques

Discussions

\section{References}

Amato, U., Masiello, G., Serio, C., and Viggiano, M.: The $\sigma$-IASI code for the calculation of infrared atmospheric radiance and its derivatives, Environmental Modelling \& Software, 17, 651-667, https://doi.org/10.1016/s1364-8152(02)00027-0, 2002.

Bianchini, G. and Palchetti, L.: Technical Note: REFIR-PAD level 1 data analysis and performance characterization, Atmospheric Chemistry and Physics, 8, 3817-3826, https://doi.org/10.5194/acp-8-3817-2008, 2008.

Bianchini, G., Carli, B., Cortesi, U., Del Bianco, S., Gai, M., and Palchetti, L.: Test of far-infrared atmospheric spectroscopy using wide-band balloon-borne measurements of the upwelling radiance, Journal of Quantitative Spectroscopy and Radiative Transfer, 109, 1030-1042, https://doi.org/https://doi.org/10.1016/j.jqsrt.2007.11.010, spectroscopy and Radiative Transfer in Planetary Atmospheres, 2008.

Bianchini, G., Palchetti, L., and Carli, B.: Vectorial combination of signals in Fourier transform spectroscopy, Infrared Physics \& Technology, 52, 19-21, https://doi.org/https://doi.org/10.1016/j.infrared.2008.09.004, 2009.

Bianchini, G., Castagnoli, F., Di Natale, G., and Palchetti, L.: A Fourier transform spectroradiometer for ground-based remote sensing of the atmospheric downwelling long-wave radiance, Atmospheric Measurement Techniques, 12, 619-635, https://doi.org/10.5194/amt-12-619$2019,2019$.

Bozzo, A., Maestri, T., Rizzi, R., and Tosi, E.: Parameterization of single scattering properties of mid-latitude cirrus clouds for fast radiative transfer models using particle mixtures, Geophysical Research Letters, 35, https://doi.org/10.1029/2008g1034695, 2008.

Brindley, H. E. and Harries, J. E.: The impact of far i.r. absorption on clear sky greenhouse forcing: sensitivity studies at high spectral resolution, Journal of Quantitative Spectroscopy and Radiative Transfer, 60, 151-180, https://doi.org/10.1016/s0022-4073(97)00152-0, 1998.

Carli, B., Bazzini, G., Castelli, E., Cecchi-Pestellini, C., Del Bianco, S., Dinelli, B., Gai, M., Magnani, L., Ridolfi, M., and Santurri, L.: MARC: A code for the retrieval of atmospheric parameters from millimeter-wave limb measurements, Journal of Quantitative Spectroscopy and Radiative Transfer, 105, 476-491, https://doi.org/https://doi.org/10.1016/j.jqsrt.2006.11.011, 2007.

Chahine, M. T., Pagano, T. S., Aumann, H. H., Atlas, R., Barnet, C., Blaisdell, J., Chen, L., Divakarla, M., Fetzer, E. J., Goldberg, M., Gautier, C., Granger, S., Hannon, S., Irion, F. W., Kakar, R., Kalnay, E., Lambrigtsen, B. H., Lee, S.-Y., Marshall, J. L., Mcmillan, W. W., Mcmillin, L., Olsen, E. T., Revercomb, H., Rosenkranz, P., Smith, W. L., Staelin, D., Strow, L. L., Susskind, J., Tobin, D., Wolf, W., and Zhou, L.: AIRS, Bulletin of the American Meteorological Society, 87, 911-926, https://doi.org/10.1175/bams-87-7-911, 2006.

Chen, X., Huang, X., and Flanner, M. G.: Sensitivity of modeled far-IR radiation budgets in polar continents to treatments of snow surface and ice cloud radiative properties, Geophysical Research Letters, 41, 6530-6537, https://doi.org/10.1002/2014g1061216, 2014.

Clough, S., Shephard, M., Mlawer, E., Delamere, J., Iacono, M., Cady-Pereira, K., Boukabara, S., and Brown, P.: Atmospheric radiative transfer modeling: a summary of the AER codes, Journal of Quantitative Spectroscopy and Radiative Transfer, 91, 233-244, https://doi.org/https://doi.org/10.1016/j.jqsrt.2004.05.058, 2005.

Clough, S. A. and Iacono, M. J.: Line-by-line calculation of atmospheric fluxes and cooling rates: 2. Application to carbon dioxide, ozone, methane, nitrous oxide and the halocarbons, Journal of Geophysical Research, 100, 16519, https://doi.org/10.1029/95jd01386, 1995.

Clough, S. A., Iacono, M. J., and Moncet, J.-L.: Line-by-line calculations of atmospheric fluxes and cooling rates: Application to water vapor, Journal of Geophysical Research: Atmospheres, 97, 15 761-15 785, https://doi.org/https://doi.org/10.1029/92JD01419, 1992.

Cortesi, U., Bianco, S. D., Gai, M., Laurenza, L., Ceccherini, S., Carli, B., Barbara, F., and Buchwitz, M.: Sensitivity analysis and application of KLIMA algorithm to GOSAT and OCO validation - KLIMA-IASI, Final Report of Project ESA-ESRIN/Contract n. 21612/08/I-OL, Tech. Rep. IFAC-TSRR, IFAC-CNR, 2014. 
https://doi.org/10.5194/amt-2021-196

Preprint. Discussion started: 2 September 2021

(c) Author(s) 2021. CC BY 4.0 License.
Atmospheric

Measurement

Techniques

Discussions

Cossich, W., Maestri, T., Magurno, D., Martinazzo, M., Natale, G. D., Palchetti, L., Bianchini, G., and Guasta, M. D.: Ice and Mixed-Phase Cloud Statistics on Antarctic Plateau, https://doi.org/10.5194/acp-2021-97, 2021.

Di Natale, G., Palchetti, L., Bianchini, G., and Del Guasta, M.: Simultaneous retrieval of water vapour, temperature and cirrus clouds properties from measurements of far infrared spectral radiance over the Antarctic Plateau, Atmospheric Measurement Techniques, 10, 825-837, https://doi.org/10.5194/amt-10-825-2017, 2017.

Di Natale, G., Bianchini, G., Del Guasta, M., Ridolfi, M., Maestri, T., Cossich, W., Magurno, D., and Palchetti, L.: Characterization of the Far Infrared Properties and Radiative Forcing of Antarctic Ice and Water Clouds Exploiting the Spectrometer-LiDAR Synergy, Remote Sensing, 12, 3574, https://doi.org/10.3390/rs12213574, 2020.

Di Natale, G., Palchetti, L., Bianchini, G., and Ridolfi, M.: The two-stream $\delta$-Eddington approximation to simulate the far infrared Earth spectrum for the simultaneous atmospheric and cloud retrieval, Journal of Quantitative Spectroscopy and Radiative Transfer, 246, 106927, https://doi.org/https://doi.org/10.1016/j.jqsrt.2020.106927, 2020.

Eremenko, M., Sgheri, L., Ridolfi, M., Cuesta, J., Costantino, L., Sellitto, P., and Dufour, G.: Tropospheric ozone retrieval from thermal infrared nadir satellite measurements: Towards more adaptability of the constraint using a self-adapting regularization, Journal of Quantitative Spectroscopy and Radiative Transfer, 238, 106 577, https://doi.org/https://doi.org/10.1016/j.jqsrt.2019.106577, 2019.

ESA: Report for Mission Selection: FORUM, Tech. Rep. ESA-EOPSM-FORM-RP-3549, European Space Agency,, 2019.

Feldman, D. R., Collins, W. D., Pincus, R., Huang, X., and Chen, X.: Far-infrared surface emissivity and climate, Proceedings of the National Academy of Sciences, 111, 16297-16302, https://doi.org/10.1073/pnas.1413640111, 2014.

Han, Y., Revercomb, H., Cromp, M., Gu, D., Johnson, D., Mooney, D., Scott, D., Strow, L., Bingham, G., Borg, L., Chen, Y., DeSlover, D., Esplin, M., Hagan, D., Jin, X., Knuteson, R., Motteler, H., Predina, J., Suwinski, L., Taylor, J., Tobin, D., Tremblay, D., Wang, C., Wang, L., Wang, L., and Zavyalov, V.: Suomi NPP CrIS measurements, sensor data record algorithm, calibration and validation activities, and record data quality, Journal of Geophysical Research: Atmospheres, 118, 12,734-12,748, https://doi.org/10.1002/2013jd020344, 2013.

Harries, J., Carli, B., Rizzi, R., Serio, C., Mlynczak, M., Palchetti, L., Maestri, T., Brindley, H., and Masiello, G.: The Far-infrared Earth, Reviews of Geophysics, 46, https://doi.org/https://doi.org/10.1029/2007RG000233, 2008.

Hersbach, H., Bell, B., Berrisford, P., Hirahara, S., Horányi, A., Muñoz-Sabater, J., Nicolas, J., Peubey, C., Radu, R., Schepers, D., Simmons, A., Soci, C., Abdalla, S., Abellan, X., Balsamo, G., Bechtold, P., Biavati, G., Bidlot, J., Bonavita, M., De Chiara, G., Dahlgren, P., Dee, D., Diamantakis, M., Dragani, R., Flemming, J., Forbes, R., Fuentes, M., Geer, A., Haimberger, L., Healy, S., Hogan, R. J., Hólm, E., Janisková, M., Keeley, S., Laloyaux, P., Lopez, P., Lupu, C., Radnoti, G., de Rosnay, P., Rozum, I., Vamborg, F., Villaume, S., and Thépaut, J.-N.: The ERA5 global reanalysis, Quarterly Journal of the Royal Meteorological Society, 146, 1999-2049, https://doi.org/https://doi.org/10.1002/qj.3803, 2020.

Hilton, F., Armante, R., August, T., Barnet, C., Bouchard, A., Camy-Peyret, C., Capelle, V., Clarisse, L., Clerbaux, C., Coheur, P.-F., Collard, A., Crevoisier, C., Dufour, G., Edwards, D., Faijan, F., Fourrié, N., Gambacorta, A., Goldberg, M., Guidard, V., Hurtmans, D., Illingworth, S., Jacquinet-Husson, N., Kerzenmacher, T., Klaes, D., Lavanant, L., Masiello, G., Matricardi, M., McNally, A., Newman, S., Pavelin, E., Payan, S., Péquignot, E., Peyridieu, S., Phulpin, T., Remedios, J., Schlüssel, P., Serio, C., Strow, L., Stubenrauch, C., Taylor, J., Tobin, D., Wolf, W., and Zhou, D.: Hyperspectral Earth Observation from IASI: Five Years of Accomplishments, Bulletin of the American Meteorological Society, 93, 347-370, https://doi.org/10.1175/bams-d-11-00027.1, 2012.

Huang, X., Chen, X., Zhou, D. K., and Liu, X.: An Observationally Based Global Band-by-Band Surface Emissivity Dataset for Climate and Weather Simulations, Journal of the Atmospheric Sciences, 73, 3541 - 3555, https://doi.org/10.1175/JAS-D-15-0355.1, 2016. 
https://doi.org/10.5194/amt-2021-196

Preprint. Discussion started: 2 September 2021

(C) Author(s) 2021. CC BY 4.0 License.
Atmospheric

Measurement

Techniques

Discussions

IPCC: Climate Change 2021: The Physical Science Basis. Contribution of Working Group I to the Sixth Assessment Report of the Intergovernmental Panel on Climate Change, Cambridge University Press, 2021.

Liou, K. and Yang, P.: Light scattering by ice crystals : fundamentals and applications, Cambridge University Press, Cambridge, 2016.

Liuzzi, G., Masiello, G., Serio, C., Palchetti, L., and Bianchini, G.: Validation of H2O continuum absorption models in the wave number range 180-600 cm-1 with atmospheric emitted spectral radiance measured at the Antarctica Dome-C site, Opt. Express, 22, 1678416801, https://doi.org/10.1364/OE.22.016784, 2014.

Maestri, T., Arosio, C., Rizzi, R., Palchetti, L., Bianchini, G., and Del Guasta, M.: Antarctic Ice Cloud Identification and Properties Using Downwelling Spectral Radiance From 100 to 1,400 cm-1, Journal of Geophysical Research: Atmospheres, 124, 4761-4781, https://doi.org/https://doi.org/10.1029/2018JD029205, 2019a.

Maestri, T., Cossich, W., and Sbrolli, I.: Cloud identification and classification from high spectral resolution data in the far infrared and mid-infrared, Atmospheric Measurement Techniques, 12, 3521-3540, https://doi.org/10.5194/amt-12-3521-2019, 2019b.

Magurno, D., Cossich, W., Maestri, T., Bantges, R., Brindley, H., Fox, S., Harlow, C., Murray, J., Pickering, J., Warwick, L., and Oetjen, H.: Cirrus Cloud Identification from Airborne Far-Infrared and Mid-Infrared Spectra, Remote Sensing, 12, https://doi.org/10.3390/rs12132097, 2020.

Martin, G. M., Johnson, D. W., and Spice, A.: The Measurement and Parameterization of Effective Radius of Droplets in Warm Stratocumulus Clouds, Journal of Atmospheric Sciences, 51, 1823 - 1842, https://doi.org/10.1175/1520-0469(1994)051<1823:TMAPOE>2.0.CO;2, 1994.

Masiello, G. and Serio, C.: Simultaneous physical retrieval of surface emissivity spectrum and atmospheric parameters from infrared atmospheric sounder interferometer spectral radiances, Appl. Opt., 52, 2428-2446, https://doi.org/10.1364/AO.52.002428, 2013.

Mlawer, E. J., Turner, D. D., Paine, S. N., Palchetti, L., Bianchini, G., Payne, V. H., Cady-Pereira, K. E., Pernak, R. L., Alvarado, M. J., Gombos, D., Delamere, J. S., Mlynczak, M. G., and Mast, J. C.: Analysis of Water Vapor Absorption in the Far-Infrared and Submillimeter Regions Using Surface Radiometric Measurements From Extremely Dry Locations, Journal of Geophysical Research: Atmospheres, 124, 8134-8160, https://doi.org/https://doi.org/10.1029/2018JD029508, 2019.

Palchetti, L., Bianchini, G., Natale, G. D., and Guasta, M. D.: Far-Infrared Radiative Properties of Water Vapor and Clouds in Antarctica, Bulletin of the American Meteorological Society, 96, 1505 - 1518, https://doi.org/10.1175/BAMS-D-13-00286.1, 2015.

Palchetti, L., Di Natale, G., and Bianchini, G.: Remote sensing of cirrus cloud microphysical properties using spectral measurements over the full range of their thermal emission, Journal of Geophysical Research: Atmospheres, 121, 10,804-10,819, https://doi.org/https://doi.org/10.1002/2016JD025162, 2016.

Palchetti, L., Brindley, H., Bantges, R., Buehler, S. A., Camy-Peyret, C., Carli, B., Cortesi, U., Del Bianco, S., Di Natale, G., Dinelli, B. M., Feldman, D., Huang, X. L., C. Labonnote, L., Libois, Q., Maestri, T., Mlynczak, M. G., Murray, J. E., Oetjen, H., Ridolfi, M., Riese, M., Russell, J., Saunders, R., and Serio, C.: FORUM: unique far-infrared satellite observations to better understand how Earth radiates energy to space, Bulletin of the American Meteorological Society, pp. 1-52, https://doi.org/10.1175/BAMS-D-19-0322.1, 2020.

Paul, M., Aires, F., Prigent, C., Trigo, I. F., and Bernardo, F.: An innovative physical scheme to retrieve simultaneously surface temperature and emissivities using high spectral infrared observations from IASI, Journal of Geophysical Research: Atmospheres, 117, https://doi.org/https://doi.org/10.1029/2011JD017296, 2012.

Remedios, J. J., Leigh, R. J., Waterfall, A. M., Moore, D. P., Sembhi, H., Parkes, I., Greenhough, J., Chipperfield, M. P., and Hauglustaine, D.: MIPAS reference atmospheres and comparisons to V4.61/V4.62 MIPAS level 2 geophysical data sets, Atmospheric Chemistry and Physics Discussions, 7, 9973-10 017, https://doi.org/10.5194/acpd-7-9973-2007, 2007. 
https://doi.org/10.5194/amt-2021-196

Preprint. Discussion started: 2 September 2021

(C) Author(s) 2021. CC BY 4.0 License.
Atmospheric

Measurement

Techniques

Discussions

Ridolfi, M. and Sgheri, L.: Iterative approach to self-adapting and altitude-dependent regularization for atmospheric profile retrievals, Opt. Express, 19, 26 696-26709, https://doi.org/10.1364/OE.19.026696, 2011.

Ridolfi, M., Del Bianco, S., Di Roma, A., Castelli, E., Belotti, C., Dandini, P., Di Natale, G., Dinelli, B. M., C.-Labonnote, L., and Palchetti, L.: FORUM Earth Explorer 9: Characteristics of Level 2 Products and Synergies with IASI-NG, Remote Sensing, 12, https://doi.org/10.3390/rs12091496, 2020.

Rodgers, C. D.: Inverse Methods for Atmospheric Sounding, WORLD SCIENTIFIC, https://doi.org/10.1142/3171, 2000.

Saito, M., Yang, P., Huang, X., Brindley, H. E., Mlynczak, M. G., and Kahn, B. H.: Spaceborne Middle- and Far-Infrared Observations Improving Nighttime Ice Cloud Property Retrievals, Geophysical Research Letters, 47, https://doi.org/10.1029/2020gl087491, 2020.

Serio, C., Masiello, G., Esposito, F., Girolamo, P. D., Iorio, T. D., Palchetti, L., Bianchini, G., Muscari, G., Pavese, G., Rizzi, R., Carli, B., and Cuomo, V.: Retrieval of foreign-broadened water vapor continuum coefficients from emitted spectral radiance in the $\mathrm{H} \_2 \mathrm{O}$ rotational band from 240 to $590 \mathrm{~cm}^{\wedge}-1$, Optics Express, 16, 15 816, https://doi.org/10.1364/oe.16.015816, 2008.

Sgheri, L. and Castelli, E.: Speeding up the DISORT solver: mathematical approach and application to radiance simulations of FORUM, in: ESA ATMOS Conference, Salzburg, 2018.

Sgheri, L., Raspollini, P., and Ridolfi, M.: Auto-adaptive Tikhonov regularization of water vapor profiles: application to FORUM measurements, Applicable Analysis, 0, 1-11, https://doi.org/10.1080/00036811.2020.1751825, 2020.

Sinha, A. and Harries, J. E.: Water vapour and greenhouse trapping: The role of far infrared absorption, Geophysical Research Letters, 22, 2147-2150, https://doi.org/10.1029/95g101891, 1995.

Stengel, M., Stapelberg, S., Sus, O., Schlundt, C., Poulsen, C., Thomas, G., Christensen, M., Carbajal Henken, C., Preusker, R., Fischer, J., Devasthale, A., Willén, U., Karlsson, K.-G., McGarragh, G. R., Proud, S., Povey, A. C., Grainger, R. G., Meirink, J. F., Feofilov, A., Bennartz, R., Bojanowski, J. S., and Hollmann, R.: Cloud property datasets retrieved from AVHRR, MODIS, AATSR and MERIS in the framework of the Cloud_cci project, Earth System Science Data, 9, 881-904, https://doi.org/10.5194/essd-9-881-2017, 2017.

Sun, Z.: Reply to comments by Greg M. McFarquhar on 'Parametrization of effective sizes of cirrus-cloud particles and its verification against observations'. (October B, 1999, 125, 3037-3055), Quarterly Journal of the Royal Meteorological Society, 127, 267-271, https://doi.org/https://doi.org/10.1002/qj.49712757116, 2001.

Sun, Z. and Rikus, L.: Parametrization of effective sizes of cirrus-cloud particles and its verification against observations, Quarterly Journal of the Royal Meteorological Society, 125, 3037-3055, https://doi.org/https://doi.org/10.1002/qj.49712556012, 1999.

Sánchez-Nogales, M.: Earth Observation Mission CFI Software GENERAL SOFTWARE USER MANUAL, DEIMOS, ESA, 2020.

Turner, D. D., Ackerman, S. A., Baum, B. A., Revercomb, H. E., and Yang, P.: Cloud Phase Determination Using Ground-Based AERI Observations at SHEBA, Journal of Applied Meteorology, 42, 701 - 715, https://doi.org/10.1175/15200450(2003)042<0701:CPDUGA>2.0.CO;2, 2003.

Veglio, P. and Maestri, T.: Statistics of vertical backscatter profiles of cirrus clouds, Atmospheric Chemistry and Physics, 11, 12 925-12943, https://doi.org/10.5194/acp-11-12925-2011, 2011.

Wessel, P. and Smith, W. H. F.: A global, self-consistent, hierarchical, high-resolution shoreline database, Journal of Geophysical Research: Solid Earth, 101, 8741-8743, https://doi.org/https://doi.org/10.1029/96JB00104, 1996.

Wielicki, B. A., Young, D. F., Mlynczak, M. G., Thome, K. J., Leroy, S., Corliss, J., Anderson, J. G., Ao, C. O., Bantges, R., Best, F., Bowman, K., Brindley, H., Butler, J. J., Collins, W., Dykema, J. A., Doelling, D. R., Feldman, D. R., Fox, N., Huang, X., Holz, R., Huang, Y., Jin, Z., Jennings, D., Johnson, D. G., Jucks, K., Kato, S., Kirk-Davidoff, D. B., Knuteson, R., Kopp, G., Kratz, D. P., Liu, X., Lukashin, C., Mannucci, A. J., Phojanamongkolkij, N., Pilewskie, P., Ramaswamy, V., Revercomb, H., Rice, J., Roberts, Y., Roithmayr, C. M., Rose, 
https://doi.org/10.5194/amt-2021-196

Preprint. Discussion started: 2 September 2021

(c) Author(s) 2021. CC BY 4.0 License.

(c) (1)

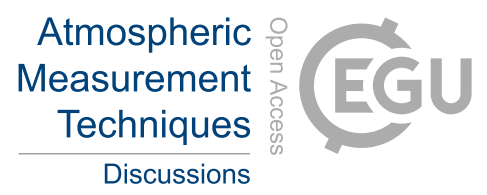

F., Sandford, S., Shirley, E. L., Smith, W. L., Soden, B., Speth, P. W., Sun, W., Taylor, P. C., Tobin, D., and Xiong, X.: Achieving Climate Change Absolute Accuracy in Orbit, Bulletin of the American Meteorological Society, 94, 1519-1539, https://doi.org/10.1175/BAMS-D12-00149.1, publisher: American Meteorological Society Section: Bulletin of the American Meteorological Society, 2013.

Yang, P., Bi, L., Baum, B. A., Liou, K.-N., Kattawar, G. W., Mishchenko, M. I., and Cole, B.: Spectrally Consistent Scattering, Absorption, and Polarization Properties of Atmospheric Ice Crystals at Wavelengths from 0.2 to $100 \mu \mathrm{m}$, Journal of the Atmospheric Sciences, 70,330 - 347, https://doi.org/10.1175/JAS-D-12-039.1, 2013. 